\title{
Coupled Processes in Charged Porous Media: From Theory to Applications
}

\author{
Vahid Joekar-Niasar ${ }^{1}$ (D) Lynn Schreyer ${ }^{2} \cdot$ Majid Sedighi $^{3} \cdot$ Matteo Icardi $^{4}$. \\ Jacques Huyghe ${ }^{5}$
}

Received: 14 August 2018 / Accepted: 26 February 2019 / Published online: 12 March 2019

(c) The Author(s) 2019

\begin{abstract}
Charged porous media are pervasive, and modeling such systems is mathematically and computationally challenging due to the highly coupled hydrodynamic and electrochemical interactions caused by the presence of charged solid surfaces, ions in the fluid, and chemical reactions between the ions in the fluid and the solid surface. In addition to the microscopic physics, applied external potentials, such as hydrodynamic, electrical, and chemical potential gradients, control the macroscopic dynamics of the system. This paper aims to give fresh overview of modeling pore-scale and Darcy-scale coupled processes for different applications. At the microscale, fundamental microscopic concepts and corresponding mass and momentum balance equations for charged porous media are presented. Given the highly coupled nonlinear physiochemical processes in charged porous media as well as the huge discrepancy in length scales of these physiochemical phenomena versus the application, numerical simulation of these processes at the Darcy scale is even more challenging than the direct pore-scale simulation of multiphase flow in porous media. Thus, upscaling the microscopic processes up to the Darcy scale is essential and highly required for large-scale applications. Hence, we provide and discuss Darcy-scale porous medium theories obtained using the hybrid mixture theory and homogenization along with their corresponding assumptions. Then, application of these theoretical developments in clays, batteries, enhanced oil recovery, and biological systems is discussed.
\end{abstract}

Keywords Porous media · Electrical double layer · Mixture theory · Electrokinetic . Charged surface $\cdot$ Coupled processes

\Vahid Joekar-Niasar

vahid.niasar@manchester.ac.uk

1 School of Chemical Engineering and Analytical Science, University of Manchester, Manchester, UK

2 Department of Mathematics and Statistics, Washington State University, Pullman, WA, USA

3 School of Mechanical, Aerospace and Civil Engineering, University of Manchester, Manchester, UK

4 School of Mathematical Sciences, University of Nottingham, Nottingham, UK

5 Bernal Institute, University of Limerick, Limerick, Ireland 


\section{Introduction}

Fluid flow, chemical transport, and deformation in charged porous media are important topics studied in many engineering applications and natural phenomena, where the electrostatic or electrokinetic forces due to the charged solid surfaces become important. Because electrical forces (due to the charged particle surfaces) are considerable at very small length scales (nanometer), these forces are dominant in porous media that have large surface-to-volume ratios or very small pore sizes. All fundamental phenomena in porous media such as singlephase and two-phase flow hydrodynamics, solute transport, and deformation can be highly influenced by charged surfaces, which are not included in the classical Darcy-scale flow and deformation theory.

Clayey soils are a notable example of porous media, where flow, transport, and deformation are strongly influenced by the charged surfaces due to a small pore size distribution and large surface area-to-volume ratio. Examples of the many different applications that take advantage of the charged porous media include remediation of clay-rich soils using electroosmosis (Kirby 2010), and slope mechanical stabilization using electroosmosis. In other fields, charged porous media are used to deliver drugs into a body using electrophoresis, enhance oil recovery by tuning the ionic composition of brine in an oil reservoir (Joekar-Niasar and Mahani 2016; Aziz et al. 2018), mix and separate in lab-on-chip applications (Guijt et al. 2001), and stabilize liquid foam (Bergeron 1999; Karraker and Radke 2002). Also surface charged properties of soils and colloids determine the transport and retention of colloids in porous media (Norde and Lyklema 1978; Naidu et al. 1994). In all these applications, the charged surface of biological, rock, sand, clay, or chemical engineering system is utilized to meet the objectives of the specific applications. Although these geological, biological, or industrial systems are very different, they share fundamentals associated with flow and transport in charged porous media.

The main purpose of this paper is to present how the surface charged properties and ionic solutions should be incorporated into the modeling of fundamental processes in porous media (e.g., flow hydrodynamics, solute transport, and deformation) at the micro- and macroscale. To our understanding, in the field of porous media there is no comprehensive overview that provides the basics of charged porous media and their incorporation in the Darcy-scale models. This paper aims to develop a bridge between the pore-scale and continuum-scale physics and upscaling the complex nonlinear physics in the Darcy-scale models. After introducing the theoretical framework for modeling pore-scale and continuum-scale phenomena, some notable examples of applications of charged porous media such as diffusion in clays, batteries, and biological systems, and enhanced oil recovery are presented to illustrate the importance of these fundamentals in different industrial, biological, and geology applications. We conclude the paper with a brief description of notable challenges at micro- and macroscale from theoretical and application perspectives.

\section{Microscale Description of Physical and Chemical Processes in Charged Porous Media}

The interaction between charged interfaces (such as what occurs in colloid-colloid interaction, thin films, and lamella stability) is mainly caused by van der Waals and electrostatic forces. The classical understanding of forces due to charged surfaces is commonly based on the DLVO theory, named after Boris Derjaguin and Lev Landau, Evert Verwey, and Theodor 
Overbeek (Ohshima 2012; Trefalt et al. 2016; Mikelonis et al. 2016). This theory enables the calculation of the net interaction forces (and alternatively pressures) due to the van der Waals forces and Coulombic (entropic) forces at a thermodynamically equilibrium state. Although there are extensions to the DLVO theory (van Oss et al. 1990; Grasso et al. 2002), the DLVO theory is still the dominant theory in practice, and we restrict ourselves to presenting this theory.

\subsection{Electrical Double Layer}

Many natural and synthetic materials have charged surfaces (e.g., clay, charged nanoparticles, polymers) due to the electron imbalance of their molecular structures. The charged surface produces an electric field, which attracts counterions (dissolved ions from a salt compound, e.g., $\mathrm{NaCl}$, in water, that have the opposite charge of the surface) in an ionic solution or electrolyte. The layer comprising surface charges and counterions is called an "electric double layer."

The simplest model for an electrical double layer was conceptualized as a layer of counterions bound directly to the surface, neutralizing the surface charges. The layer was referred to as the Helmholtz layer. If this layer fully neutralized the surface charge, the electric field at a distance of a molecular layer (in angstrom size) from the surface would be zero, which is not the case. Later, Gouy and Chapman included the thermal motion of ions and proposed the diffuse layer. In 1924 Stern suggested that by combining the Helmholtz model and the Gouy-Chapman model, the electrical capacity of charged systems in low and high charged conditions was explained. In Stern's model, some ions adhere to the electrode as suggested by Helmholtz (hydrodynamically not movable), giving an internal Stern layer, while some other ions would form a Gouy-Chapman diffuse layer, as shown in Fig. 1. The electrical potential measured at the shear plane (also called the slipping plane), roughly at the interface between the Stern layer and diffuse layer, is referred to as the zeta potential $(\zeta)$. The zeta potential is one of the key measurable quantities that characterizes the electrical potential of an interface at a given $\mathrm{pH}$ and ionic strength (Hunter 1988; Kirby and Hasselbrink 2004).

The distribution of ions in the vicinity of the surface is theoretically defined using the Boltzmann distribution normal to the surface. The Boltzmann distribution can be derived using statistical thermodynamics (Hill 1960), and it determines the probability of microscopic states, $P(W)$, given the energy $(W)$, as follows:

$$
P(W) \propto \exp \left(-\frac{W}{k_{\mathrm{B}} T}\right),
$$

where $T$ is the absolute temperature, $k_{\mathrm{B}}$ denotes the Boltzmann constant defined as $R / N_{a}$, where $R$ is the universal gas constant and $N_{a}$ is Avogadro's number. See Nomenclature "Appendix" for definitions of all variables and their units.

The work required to displace an ion from a zero electric potential to a given potential at a specific position is equal to $z_{i} e \psi$, where $z_{i}$ is the valence for species $i$, positive for cations such as $\mathrm{Na}^{+}$and negative for anions such as $\mathrm{Cl}^{-}, e$ is the elementary charge, and $\psi$ is the electric potential. Thus, the probability of finding an ion at position $x$ can be written as

$$
P(\psi) \propto \exp \left(-\frac{z_{i} e \psi}{k_{\mathrm{B}} T}\right)
$$

and this is called the Boltzmann distribution. 
The Boltzmann distribution provides an equilibrium ionic concentration as a function of the electric potential. For a bulk solution, where the electric potential $\psi$ is equal to zero, $P(0)=1$. The ratio of $P(\psi) / P(0)$ is well approximated by the ratio of molar concentrations of species $i$ to that for the species in a bulk solution where the electric potential is zero, $c_{i} / c_{b_{i}}$. Thus, we can write

$$
c_{i}=c_{b_{i}} \exp \left(-\frac{z_{i} e \psi}{k_{\mathrm{B}} T}\right) .
$$

Note that the anions and cations will appear together in the bulk phase because, by definition, it has zero electric potential and zero net charge density $\left(\sum z_{i} c_{b_{i}}=0\right)$. Thus, the anion and cation concentrations are the same for a homovalent electrolyte.

In Eq. (3), the sign of $\psi$ is controlled by the sign of the boundary condition while the $z_{i}$ sign is ion-dependent. Thus, the concentrations of the co-ions (with respect to the sign of the surface charge) in the diffuse layer will always be smaller than (or equal to) the bulk concentration $\left(c_{b_{i}}\right)$ and the concentration of the counterions in the diffuse layer will be larger than $c_{b_{i}}$.

The distributions of cations and anions of a symmetric $z$-valent electrolyte (e.g., $\mathrm{NaCl}$ or $\mathrm{CaSO}_{4}$ ) in the vicinity of a negatively charged surface are schematically shown in Fig. 1, which shows the accumulation of positive ions closer to the surface compared to the negative ions. The length scale of the electric potential decay from the solid surface is described by what is called the Debye length, $\lambda_{D}$, which is inversely proportional to the square root of the ionic strength, $\Gamma$, written as (Lyklema 1995)

$$
\begin{aligned}
\lambda_{D} & =\left(\frac{\epsilon k_{\mathrm{B}} T}{2 e^{2} \Gamma}\right)^{0.5} \\
\Gamma & =0.5 \sum_{i} c_{b_{i}} N_{a} z_{i}^{2},
\end{aligned}
$$

where $\epsilon$ is permittivity (Farad $\mathrm{m}^{-1}$ or $\mathrm{CV}^{-1} \mathrm{~m}^{-1}$ ), a property of a material representing its ability to store electrical energy.

\subsection{Electric Field in the Diffuse Layer}

For a solid surface with the negative charge density of $\sigma$, more positive ions (cations) accumulate next to the surface to balance the solid surface charge (electroneutrality law). The net charge concentration at the position of $x$ is introduced as $\rho_{e}(x)=e N_{a}\left(\sum z_{i}^{+} c_{i}^{+}(x)-\right.$ $\left.\sum z_{i}^{-} c_{i}^{-}(x)\right)$.

Considering the surface charge density of $\sigma$ and to guarantee electroneutrality within the diffuse layer, we write $\sigma+\int_{0}^{\infty} \rho_{e}(x) \mathrm{d} x=0, x$ is the coordinate normal to the surface. Note that for an isolated surface, the surface charge density can be neutralized at a small distance (few Debye length). Within this distance, an electric field is created which strongly varies at the pore scale with the surface charge density as well as the electrolyte concentration.

The electric field within the diffuse layer $\psi(\mathrm{V})$ is described by the Poisson equation,

$$
\nabla^{2} \psi=-\frac{\rho_{e}}{\epsilon}
$$


(a)

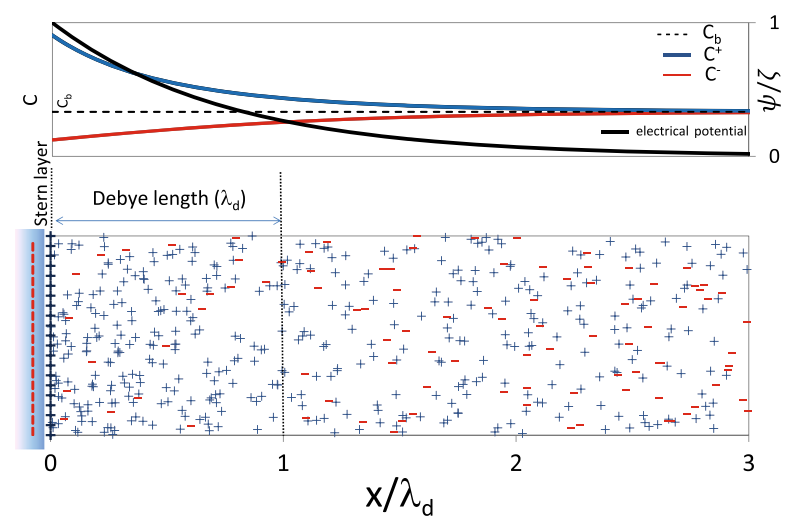

(b)

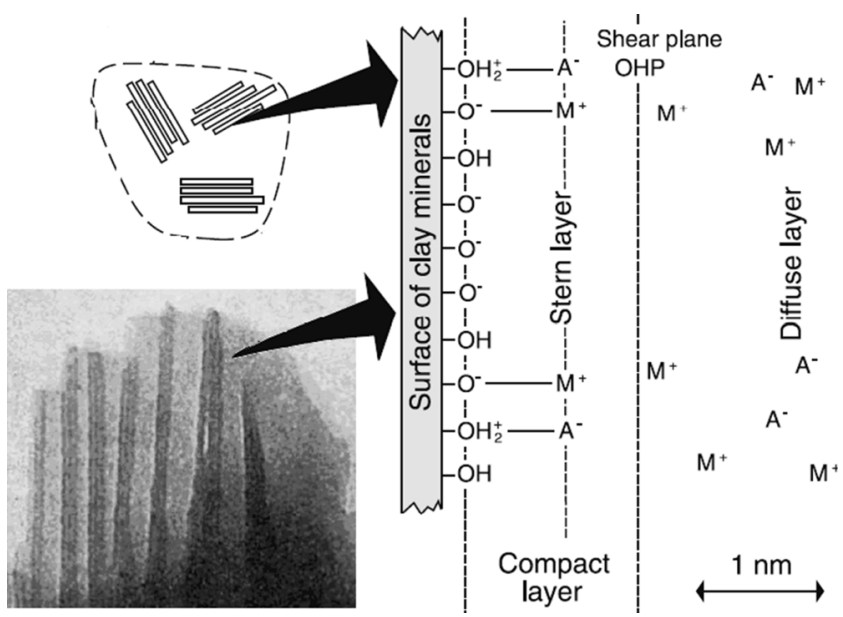

Fig. 1 a Schematic presentation of electrical double layer. For a negatively charged surface, there will be a fixed layer of positive ions referred to as the Stern layer neighboring the diffuse layer, where the distribution of ions will follow the Boltzmann distribution. The length scale for the decay of the electrical potential is referred to as the Debye length $\lambda_{d}$. The distribution of cations (blue), anions (red), and electrical potential (black) is shown in Fig. 1a. The electrical potential has been scaled to the zeta potential, b the electrical diffuse layer and its components at the clay basal surface in the case of a binary monovalent electrolyte, $\mathrm{M}^{+}$represents the cations (e.g., $\mathrm{Na}^{+}$) and $\mathrm{A}^{-}$the anions (e.g., $\mathrm{Cl}^{-}$). OHP represents the outer Helmholtz plane or shear plane (Adopted from Leroy and Revil 2004); SEM picture of sodium bentonite (Barclay and Thompson 1969)

Using the definition of net charge density and the Boltzmann distribution of ions at the equilibrium state (Eq. (3)), the Poisson-Boltzmann equation can be written as follows

$$
\nabla^{2} \psi=-\sum_{i=1}^{N} \frac{z_{i} e c_{b_{i}} N_{a}}{\epsilon} \exp \left(-\frac{z_{i} e \psi}{k_{\mathrm{B}} T}\right)
$$

Assuming only two charged species in the fluid (a cation and an anion), and a symmetric z-valent solution, the simpler Poisson-Boltzmann equation with the sinh term is obtained:

$$
\nabla^{2} \psi=2 \frac{z e c_{b} N_{a}}{\epsilon} \sinh \left(\frac{z e \psi}{k_{\mathrm{B}} T}\right)
$$


The Poisson-Boltzmann equation describes the electric field under equilibrium conditions and is not valid for non-equilibrium conditions. This is a nonlinear equation, which can be linearized if $\left|\frac{z e \psi}{k_{\mathrm{B}} T}\right| \ll 1$. Given that the maximum potential in the diffuse layer occurs at the slip plane, referred to as the zeta potential, we can write $\left|\frac{z e \zeta}{k_{\mathrm{B}} T}\right| \ll 1$. Thus, we can approximately estimate that for the zeta potential smaller than $20 \mathrm{mV}$ the linearization is valid.

By replacing the $\sinh \left(\frac{z e \psi}{k_{\mathrm{B}} T}\right)$ with $\frac{z e \psi}{k_{\mathrm{B}} T}$, Eq. (8) gives $\nabla^{2} \psi=\frac{2 z^{2} e^{2} c_{b} N_{a}}{\epsilon k_{\mathrm{B}} T} \psi$. This linearization is also called Debye-Hückel approximation. For an isolated single charged plane, the solution of the linear Poisson-Boltzmann equation is $\psi=\psi_{0} \exp \left(-\frac{z^{2} e^{2} c_{b} N_{a}}{\epsilon k_{\mathrm{B}} T} x^{2}\right)$ where $x$ is the coordinate normal to the plane. Note that the scaling factor in the exponent is the reciprocal of the squared Debye length; $\lambda_{D}^{2}=\left(\frac{z^{2} e^{2} c_{b} N_{a}}{\epsilon k_{\mathrm{B}} T}\right)^{-1}$.

\subsection{Boundary Conditions for Charged Surfaces}

To solve for either the equilibrium or non-equilibrium electrical field using either Eq. (7) or Eq. (6), boundary conditions for the potential should be defined. Three commonly used boundary conditions are: (i) constant potential, (ii) constant charge, and (iii) surface complexion models.

The constant potential boundary condition is defined as the potential measured at the slip plane and is referred to as the zeta potential $\zeta$. The slip plane is shown in Fig. 1b, and the ions positioned between the slip plane and the solid surface are assumed to be immobile. The zeta potential at the slip plane can be measured indirectly using electrophoresis and light scattering techniques, where the hydrodynamic flow is linked to the potential measured on the slip plane using some inherited hydrodynamic assumptions (Kirby and Hasselbrink 2004). It is known that the zeta potential is a strong function of $\mathrm{pH}$ and ionic strength and is not constant for all chemical conditions. Thus, a constant charge density may represent the solid interface better than the constant potential condition. The constant charge boundary condition relates the surface charge density $(\sigma)$ to the potential using $\sigma=\nabla \psi \cdot \mathbf{n}$.

However, none of the previous boundary conditions considers sorption or interaction between ions in the solution and species on the solid surface. Surface complexion models are designed to incorporate the coupling between the surface chemistry, chemical reactions for sorption and the surface charge using the stoichiometry of surface ionization reactions as well as ionization constants (Davis et al. 1978; Goldberg 1992, 2014). The most common approaches for surface complexation models are the one $\mathrm{pK}$ value model, the triple-layer model, and charge distribution model.

In the triple-layer model, as the name suggests, three layers are assumed: the diffuse layer, sorption layer, and protonation/deprotonation layer as shown in Fig. 1b. At the interface between each of the two neighboring layers, the potential and surface charge density are defined. As originally developed by Davis et al. (1978), the protonation/deprotonation of surface sites is restricted to an innermost plane (left interface in Fig. 1b) and specifically adsorbed ions are assigned to the middle plane in Fig. 1b. The last outermost plane describes the boundary for the diffuse layer (counterions extending into the bulk solution). Since the chemical reactions for sorption and stoichiometry (pK values, the equilibrium constants for chemical reactions) are incorporated in this model, it can accommodate the impact of $\mathrm{pH}$ and reactions on the surface charge density and the zeta potential (Davis et al. 1978). The charge distribution model which was developed by Hiemstra and Riemsdijk (1996) does not consider surface complexation as points, and the spatial distribution of them is also 
considered. In this model, $\mathrm{pK}$ values of different types of surface groups are estimated based on valence of cations of (hydr)oxides and electron configurations as well as the distance between the reacting surface groups.

\subsection{Microscopic Description of Fluid Flow in Charged Systems}

In cases where the fluid velocity is considerable, such as fluid flow in lamellas (related to foam instability) or thin film hydrodynamics, the fluid velocity, $\mathbf{v}$, should be calculated. The linear momentum balance equation governing liquid flow with a viscosity of $\eta$ is given by the Navier-Stokes equation supplemented with appropriate electric body forces (Hunter 1989; Revil and Linde 2006):

$$
\rho \frac{D \mathbf{u}}{D t}=-\nabla p+\eta \nabla^{2} \mathbf{v}+\nabla \cdot \underline{t}_{m},
$$

where ${ }_{=t}$. denotes the Maxwell Stress Tensor (MST), which accounts for the stress generated by electrical and magnetic fields. However, in this work, magnetic effects are considered negligible. Please note that $\nabla \cdot \underline{\boldsymbol{t}}_{m}$ can be also replaced by $\rho_{e} \boldsymbol{E}$, which is the body force applied to the ions (Israelachvili and Pashley 1982).

Assuming the electrical origin of force is due to an electric field, $\boldsymbol{E}$, that satisfies the Poisson (Eq. 6) or Poisson-Boltzmann (Eq. 8) equations, the MST including the osmotic pressure can be written as follows (Gilson et al. 1993):

$$
\underset{=}{\boldsymbol{t}}=\Pi \boldsymbol{I}+\frac{1}{2} \epsilon|E|^{2} \boldsymbol{I}-\epsilon \boldsymbol{E} \otimes \boldsymbol{E},
$$

where $\Pi$ is the osmotic pressure, representing the change in the chemical potential of the fluid due to the presence of ions in the liquid. Because, e.g., water is polarized (positive on one end, negative on the other), its affinity for being in one location over another is highly influenced by the presence of ions and this is captured by the electrochemical osmotic pressure.

The osmotic pressure at equilibrium due to the ions in the fluid phase only is given by $\Pi=2 N_{a} k_{\mathrm{B}} T c_{b}$. Given this definition, the differential osmotic pressure, $\Pi_{d}$, representing the difference between the osmotic pressure in the pore and that in the fluid's reservoir connected to the porous medium, can be defined as (Butt et al. 2013):

$$
\Pi_{d}=N_{a} k_{\mathrm{B}} T\left(c^{+}+c^{-}-2 c_{b}\right) .
$$

The differential osmotic pressure is a measure of the affinity of the liquid to be in the porous medium as opposed to the bulk phase, and this differential osmotic pressure shows the amount of the pressure required to stop movement of water molecules from the low concentration zone to the high concentration zone.

Equation (11) does not hold under non-equilibrium conditions as the distribution of ions under non-equilibrium conditions does not follow the Boltzmann distribution; $c^{ \pm} \neq$

$c_{b}^{ \pm} \exp (\mp \psi)$ (Joekar-Niasar and Mahani 2016.) However, under non-equilibrium conditions the (electrokinetic) osmotic pressure can be defined by substituting $c_{b}=c^{ \pm} \exp ( \pm \psi)$ in Eq. (11) (Revil and Linde 2006; Joekar-Niasar and Mahani 2016). This leads to the generalized form of the differential osmotic pressure which is valid under non-equilibrium and equilibrium conditions:

$$
\Pi_{d}=N_{a} k_{\mathrm{B}} T \Sigma c^{ \pm}(1-\exp ( \pm \psi))
$$

Note that since Eq. (11) deals with the differential pressure compared to the bulk fluid osmotic pressure, Eq. (12) would also indicate the electrokinetic osmotic pressure compared to the bulk fluid osmotic pressure. 
By solving Eqs. (9), (10), and (12), coupled with the mass conservation law for an incompressible fluid $\nabla \cdot \mathbf{v}=0$, the fluid pressure, $p$, can be calculated. The same sets of equations can be used for thin films to calculate pressure inside the film which is referred to as the disjoining pressure. By definition, the disjoining pressure is the pressure difference between the pressure inside the thin film and the pressure in the bulk solution. The disjoining pressure is made of two major components as delineated by the Maxwell stress tensor: electroosmotic pressure (due to local concentrations) and electrokinetic-induced pressure (due to the electric field). Of course in very small length scales (smaller than nanometer), other surface forces such as the hydration and van der Waals forces contribute to the disjoining pressure. Since Eq. (12) already includes the differential electroosmotic pressure (which converges to the chemical osmotic pressure at equilibrium) the resulting pressure, $p$, in Eq. (9) would only show the differential pressure induced purely by the electric field.

More detailed analysis of the disjoining at non-equilibrium conditions can be found in the reference (Joekar-Niasar and Mahani 2016).

\subsection{Microscopic lonic Transport in Charged Systems}

To investigate the dynamics electrical double layer and the ionic transport, the coupling between electric field (Poisson equation, which is valid under equilibrium and nonequilibrium conditions) and transport of the ions are combined. The transport of ions within the diffuse layer is governed by the Nernst-Planck equation, derived from the Smoluchowski diffusion equation (Israelachvili and Pashley 1982). It is a combination of the conservation of mass for component $i$ and a generalized Fick's equation where the concentration is replaced by the electrochemical potential, $\mu_{i}$. The electrochemical potential of the ion $i$ is the summation of the electrical and chemical potentials:

$$
\mu_{i}=e z_{i} \psi+k_{\mathrm{B}} T \ln a_{i} .
$$

$a_{i}$ denotes the chemical activity (dimensionless), which can be written as $a_{i}=\gamma_{i} c_{i} / c^{*} \cdot \gamma_{i}$ is the activity coefficient (dimensionless), $c^{*}$ is the standard concentration of a dilute solution equal to $1 \mathrm{~mol} / \mathrm{lit}$ and $\gamma_{i}$ is assumed to be 1 . Therefore, Eq. (13) can be written as

$$
\mu_{i}=e z_{i} \psi+k_{\mathrm{B}} T \ln c_{i} .
$$

The total molar flux of ion, $i$, denoted as $\mathbf{j}_{i}$, and the conservation of ions in a medium can be written as,

$$
\begin{aligned}
& \mathbf{j}_{i}=c_{i} \mathbf{v}-\frac{D_{i}}{k_{\mathrm{B}} T} c_{i} \nabla \mu_{i} \\
& \partial_{t} c_{i}+\left(\nabla \cdot \mathbf{j}_{i}+R_{i}\right)=0,
\end{aligned}
$$

where $\mathbf{v}$ is the velocity of the fluid, $R_{i}$ is the rate of desorption or adsorption of the species $i$ per unit volume, and $D_{i}$ denotes the binary diffusion coefficient for ion $i$. Note that in some charged porous media applications (e.g., batteries, compacted clays) the advective part is negligible $(\mathbf{v}=0)$. By ignoring the electrical potential, the classical convective-diffusion equation is obtained in terms of concentration. Also, the stoichiometry forces the zero net charge in chemical reactions: $\sum\left(z_{i} R_{i}\right)=0$.

When there is no external electric field, electroneutrality is an important condition that should be met in charged porous media. There are two interpretations of electroneutrality proposed by Ben-Yaakov (1972) and Lasaga (1079) as discussed by Boudreau et al. (2004b). The first interpretation is based on bulk solution charge neutrality $\left(\sum z_{i} c_{b_{i}}=0\right)$, and the 
second interpretation is based on $\sum z_{i} J_{i}=0 . J_{i}$ represents the macroscopic flux of ions flowing through a porous medium. The difference is due to the interpretation of the scale of electroneutrality, as within the electrical double layer, charge neutrality does not hold $\sum z_{i} c_{i} \neq 0$. Therefore, the question is: at what scale can charge neutrality be enforced? Within the double layer, the electrical potential is nonzero $\left(\sigma=e N_{a} \int\left(z_{i} c_{b_{i}}\right) \mathrm{d} x\right)$, but outside the double layer $\sum z_{i} c_{i}=0$. Typically, in tight porous media, where the double-layer length scale is comparable to the pore size, electroneutrality is enforced by including not only the ions in the liquid phase, but also the surface charge density.

Based on the assumption of zero total ionic transport (Boudreau et al. 2004b) (which is only acceptable if the pore size distribution is much larger than the double-layer length scale), the cross-coupling diffusion coefficient, $D_{i j}$, can be written as

$$
D_{i j}=\delta_{i j} D_{i}-\frac{z_{i} z_{j} D_{i} D_{j} c_{b_{i}}}{\sum_{k} z_{k}^{2} D_{k} c_{b_{k}}}
$$

which can be used as a general diffusion coefficient. $\delta_{i j}$ is the Dirac delta function equal to 1 for $i=j$, otherwise 0 for $i \neq j$. However, this means that if the pore size distribution is on the same size or smaller than the double-layer length scale, this cross-coupling diffusion relation $D_{i j}$ would not hold.

To summarize, the electrical potential at equilibrium can be solved using Eq. (8). However, the non-equilibrium case can be solved by combining Eqs. (6) and (16) along with Eqs. (13) and (15).

\section{Macroscopic Modeling}

Just as with non-charged porous media, equations may be upscaled using any of the same techniques, for example, volume averaging or homogenization, see, e.g., Cushman et al. (2002). By microscale we mean the scale at which one can distinguish between phases, and by macroscale we mean the scale at which one cannot distinguish between phases. We first present sample upscaled field equations, equations that are independent of the material being modeled such as the conservation of mass and momentum. We then discuss how upscaled constitutive equations can be obtained using a mixture theoretic approach, presenting macroscopic flow and diffusion equations. We then compare these results with results obtained from homogenization.

\subsection{Sample of Volume-Averaged Field Equations}

We first present a few examples of macroscopic field equations obtained using volume averaging. Within this framework, the macroscopic equations are viewed as overlaying continua, e.g., at each point in space and time there is a density of the liquid phase and the solid phase, just as in a beaker of a multicomponent fluid at each time and at each point in space there is a concentration of multiple species.

We note that since no constitutive assumptions are made, these equations are valid for all porous materials. For simplicity let us assume there are two phases, $\alpha=l$ for liquid and $\alpha=s$ for solid, and that each phase has multiple constituents, $j=1, \ldots, n$, some of which may be charged. Further, we assume that the interfaces are massless, chargeless, and no linear momentum is lost when transferred from one phase to the other. For a detailed description 
of the derivation and the complete list of all equations (in species form) see Bennethum and Cushman (2002a). A few examples are provided below:

Conservation of Mass

The macroscopic mass balance for constituent $j$ in phase $\alpha$ is

$$
\frac{D^{\alpha_{j}}\left(\varepsilon^{\alpha} \rho^{\alpha_{j}}\right)}{D t}+\varepsilon^{\alpha} \rho^{\alpha_{j}}\left(\nabla \cdot \boldsymbol{v}^{\alpha_{j}}\right)=\varepsilon^{\alpha} \rho^{\alpha_{j}} \widehat{e}_{\beta}^{\alpha_{j}}+\varepsilon^{\alpha} \rho^{\alpha_{j}} \widehat{r}^{\alpha_{j}}
$$

where $\frac{D^{\alpha_{j}}}{D t}$ is the material time derivative given by

$$
\frac{D^{\alpha_{j}}}{D t}=\frac{\partial}{\partial t}+\boldsymbol{v}^{\alpha_{j}} \cdot \nabla,
$$

$\varepsilon^{\alpha}$ is the volume fraction of phase $\alpha$, and $\widehat{e}_{\beta}^{\alpha_{j}}$ represents the net rate of mass gained by constituent $j$ in phase $\alpha$ from phase $\beta$ :

$$
\widehat{e}_{\beta}^{\alpha_{j}}=\frac{1}{\rho^{\alpha_{j}}\left|\delta V_{\alpha}\right|} \int_{\delta A_{\alpha \beta}} \rho^{j}\left(\boldsymbol{w}_{\alpha \beta}^{j}-\boldsymbol{v}^{j}\right) \cdot \boldsymbol{n}^{\alpha} \mathrm{d} a,
$$

where $\delta V_{\alpha}$ is the portion of the Representative Elementary Volume (REV) that is phase $\alpha$, $\delta A_{\alpha \beta}$ is the liquid-solid interfacial area within the $\mathrm{REV}, \rho^{j}$ is the microscopic mass density of component $j$ in phase $\alpha, \boldsymbol{n}^{j}$ is the unit normal pointing out of phase $\alpha$, and $\boldsymbol{w}_{\alpha \beta}^{j}$ is the velocity of species $j$ at interface $\alpha \beta$. We refer readers to the Nomenclature, provided in "Appendix" at the end of this paper, for definitions of the numerous variables and dimensions.

To obtain the bulk-phase counterpart, Eq. (19) is summed over all species, giving

$$
\frac{D^{\alpha}\left(\varepsilon^{\alpha} \rho^{\alpha}\right)}{D t}+\varepsilon^{\alpha} \rho^{\alpha}\left(\nabla \cdot \boldsymbol{v}^{\alpha}\right)=\varepsilon^{\alpha} \rho^{\alpha} \widehat{e}_{\beta}^{\alpha} .
$$

The net gain of mass of the bulk phase due to chemical reactions occurring in that phase must be zero, implying that:

$$
\sum_{j=1}^{N} \rho^{\alpha_{j}} \widehat{r}^{\alpha_{j}}=0 \quad \forall \alpha .
$$

Further, since the interface is assumed to be massless, no mass is lost in transferring between phases, and so we have the restrictions:

$$
\varepsilon^{\alpha} \rho^{\alpha_{j}} \widehat{e}_{\beta}^{\alpha_{j}}+\varepsilon^{\beta} \rho^{\beta_{j}} \widehat{e}_{\alpha}^{\beta_{j}}=0, \quad j=1, \ldots, N
$$

Using Eq. (21), we can rewrite Eq. (18) as

$$
\varepsilon^{\alpha} \rho^{\alpha} \frac{D^{\alpha} c^{\alpha_{j}}}{D t}+\nabla \cdot\left(\varepsilon^{\alpha} \rho^{\alpha_{j}} \boldsymbol{v}^{\alpha_{j}, \alpha}\right)=\sum_{\beta \neq \alpha} \varepsilon^{\alpha} \rho^{\alpha_{j}}\left(\widehat{e}_{\beta}^{\alpha_{j}}-\widehat{e}_{\beta}^{\alpha}\right)+\varepsilon^{\alpha} \rho^{\alpha_{j}} \widehat{r}^{\alpha_{j}} .
$$

Usually, $\rho^{\alpha_{j}}$ is considered a primary unknown and constitutive equations are needed for the rate of exchange terms $\widehat{e}_{\beta}^{\alpha_{j}}$ and $\widehat{r}^{\alpha_{j}}$. See the next section for examples.

Conservation of Charge

This equation can be derived by taking the divergence of Ampère's law and the time derivative of Gauss' Law and summing the two results. Of all Maxwell's equations, this equation is the most accepted in mixture form since each component has a well defined physical interpretation. The conservation of electric charge for species $j$ is 


$$
\frac{\partial}{\partial t}\left(\varepsilon^{\alpha} q_{e}^{\alpha_{j}}\right)+\nabla \cdot\left(\varepsilon^{\alpha} \mathcal{J}^{\alpha_{j}}+\varepsilon^{\alpha} q_{e}^{\alpha_{j}} \boldsymbol{v}^{\alpha_{j}}\right)=\varepsilon^{\alpha} \widehat{q}^{\alpha_{j}}+\varepsilon^{\alpha} \rho^{\alpha_{j}} z^{\alpha_{j}} \widehat{r}^{\alpha_{j}}+\varepsilon^{\alpha} \rho^{\alpha_{j}}\left(\widehat{Z}_{\beta}^{\alpha_{j}}+z^{\alpha_{j}} \widehat{e}_{\beta}^{\alpha_{j}}\right)
$$

where $q_{e}^{\alpha_{j}}$ is the charge density averaged over the $\alpha$-phase, $\mathcal{J}^{\alpha_{j}}$ is the upscaled free current density of constituent $j$ measured relative to species $\alpha_{j}$, and $z^{\alpha_{j}}$ is the averaged charge per unit mass (usually fixed except for in plasma). Here we have four rate of exchange terms, two within phase $\alpha$ and two between phases: $\widehat{q}^{\alpha_{j}}$ is the rate of gain of charge density due to the presence of other constituents in phase $\alpha$ but not due to chemical reactions (often assumed to be zero except for in plasma), $\varepsilon^{\alpha} \rho^{\alpha_{j}} z^{\alpha_{j} \widehat{r}^{\alpha_{j}}}$ is the rate of gain of charge density due to mass transfer (chemical reactions), $\widehat{Z}_{\beta}^{\alpha_{j}}$ is the rate of exchange of charge of constituent $j$ from phase $\beta$ to phase $\alpha$ induced by the normal component of the free current density averaged over the liquid-solid interface and a moving interface (see Bennethum and Cushman (2002a) for details), and $z^{\alpha_{j}} \hat{e}_{\beta}^{\alpha_{j}}$ is the rate of gain of charge density due to the rate of mass transfer between phases. The free current density, denoted by $\mathcal{J}^{\alpha_{j}}$, is related to the free current density relative to a fixed (Eulerian) frame of reference, $\boldsymbol{J}^{\alpha_{j}}$, by

$$
\mathcal{J}^{\alpha_{j}}=\boldsymbol{J}^{\alpha_{j}}-q_{e}^{\alpha_{j}} \boldsymbol{v}^{\alpha_{j}}
$$

Another form of this equation is obtained by subtracting out the conservation of mass to reduce redundancy, and this yields

$$
\varepsilon^{\alpha} \rho^{\alpha_{j}} \frac{D^{\alpha_{j}} z^{\alpha_{j}}}{D t}+\nabla \cdot\left(\varepsilon^{\alpha} \mathcal{J}^{\alpha_{j}}\right)=\varepsilon^{\alpha} \widehat{q}^{\alpha_{j}}+\varepsilon^{\alpha} \rho^{\alpha_{j}} \widehat{Z}_{\beta}^{\alpha_{j}},
$$

indicating that if the upscaled charge density of each component only changes due to chemical reactions, then the divergence of the free current density is (unlike for a single phase) not zero.

Summing over constituents yields

$$
\frac{\partial\left(\varepsilon^{\alpha} q_{e}^{\alpha}\right)}{\partial t}+\nabla \cdot\left(\varepsilon^{\alpha} \mathcal{J}^{\alpha}+q_{e}^{\alpha} \boldsymbol{v}^{\alpha}\right)=\varepsilon^{\alpha} \rho^{\alpha}\left(\widehat{Z}_{\beta}^{\alpha}+z^{\alpha} \widehat{e}_{\beta}^{\alpha}\right),
$$

where $\boldsymbol{J}^{\alpha}=\sum_{j=1}^{N} \boldsymbol{J}^{\alpha_{j}}, \mathcal{J}^{\alpha}=\sum_{j=1}^{N} \mathcal{J}^{\alpha_{j}}+q_{e}^{\alpha_{j}} \boldsymbol{v}^{\alpha_{j}, \alpha}$, and where the following restrictions apply

$$
\begin{aligned}
& \sum_{j=1}^{N}\left[\widehat{q}^{\alpha_{j}}+\rho^{\alpha_{j}} z^{\alpha_{j} \widehat{r}^{\alpha_{j}}}\right]=0 \quad \forall \alpha \\
& \varepsilon^{\alpha} \rho^{\alpha_{j}}\left(\widehat{Z}_{\beta}^{\alpha_{j}}+z^{\alpha_{j}} \widehat{e}_{\beta}^{\alpha_{j}}\right)+\varepsilon^{\beta} \rho^{\beta_{j}}\left(\widehat{Z}_{\alpha}^{\beta_{j}}+z^{\beta_{j}} \widehat{e}_{\alpha}^{\beta_{j}}\right)=0 \quad j=1, \ldots, N .
\end{aligned}
$$

Equation (29) states that no net charge is lost due to ion transfer or chemical reactions, and Eq. (30) states that no net charge is lost through the interface. Relating the exchange terms to their microscopic counterparts (Bennethum and Cushman 2002a), we see that

$$
\varepsilon^{\alpha} \rho^{\alpha_{j}}\left(\widehat{Z}_{\beta}^{\alpha_{j}}+z^{\alpha_{j}} \widehat{e}_{\beta}^{\alpha_{j}}\right)=\frac{1}{|\delta V|} \int_{\delta A_{\alpha \beta}}\left[q_{e}^{j}\left(\boldsymbol{w}_{\alpha \beta}^{j}-\boldsymbol{v}^{j}\right)-\mathcal{J}^{j}\right] \cdot \boldsymbol{n}^{\alpha} d a
$$

so that we see Eq. (30) corresponds precisely with the classical jump condition across a discontinuous interface (Eringen and Maugin 1990):

$$
\boldsymbol{n} \cdot\left[\left[\mathcal{J}+q_{e}(\boldsymbol{v}-\boldsymbol{w})\right]\right]=0 .
$$




\section{Conservation of Momenta}

The conservation of linear momentum for bulk phase $\alpha$, assuming that the electric field is dominant (the magnitude of the magnetic field is small relative to the magnitude of the electric field), includes the effects of the electric field and the polarization density of material $\alpha$ :

$$
\begin{aligned}
& \varepsilon^{\alpha} \rho^{\alpha} \frac{D^{\alpha} \boldsymbol{v}^{\alpha}}{D t}-\nabla \cdot\left(\varepsilon^{\alpha} \underline{\underline{t}}^{\alpha}\right)-\varepsilon^{\alpha} \rho^{\alpha}\left(\boldsymbol{g}+\boldsymbol{g}_{I}^{\alpha}\right)-\varepsilon^{\alpha} q_{e}^{\alpha} \boldsymbol{E}_{\mathrm{T}}-\varepsilon^{\alpha} \boldsymbol{P}^{\alpha} \cdot \nabla \boldsymbol{E}_{\mathrm{T}} \\
& +\frac{1}{2} \varepsilon_{0} \boldsymbol{E}_{\mathrm{T}} \cdot \boldsymbol{E}^{\alpha} \nabla \varepsilon^{\alpha}=\sum_{\beta \neq \alpha} \varepsilon^{\alpha} \rho^{\alpha} \widehat{\boldsymbol{T}}_{\beta}^{\alpha},
\end{aligned}
$$

where $\boldsymbol{g}$ is the external supply of momentum due to gravity, $\boldsymbol{g}_{I}^{\alpha}$ is the pseudo-external supply of momentum due to the difference between the product of the averages and the average of the products of $\boldsymbol{\nabla} \boldsymbol{E} \cdot \boldsymbol{E}_{j}$ and $\boldsymbol{\nabla} \boldsymbol{E}_{j} \cdot \boldsymbol{E}$ (Bennethum and Cushman 2002a), $\boldsymbol{E}_{\mathrm{T}}$ is the (total) electric field intensity generated by ions within the porous medium and externally applied, $\boldsymbol{E}^{\alpha}$ is the electric field generated by ions within phase $\alpha, \boldsymbol{P}^{\alpha}$ is the polarization density, and $\varepsilon_{0}$ is a universal constant representing the permittivity in a vacuum.

There are two forces commonly referred to that appear in the conservation of linear momentum. The Lorentz force is the force acting on a point charge due to the presence of an electric field, and in this case is denoted by $\varepsilon^{\alpha} q_{e}^{\alpha} \boldsymbol{E}_{\mathrm{T}}$, and the force resulting from the dipole moment is called the Kelvin force (Eringen and Maugin 1990). Restrictions resulting from assuming no loss of net momenta across an interface are

$$
\varepsilon^{\alpha} \rho^{\alpha_{j}}\left(\widehat{\boldsymbol{T}}_{\beta}^{\alpha_{j}}+\widehat{e}_{\beta}^{\alpha_{j}} \boldsymbol{v}^{\alpha_{j}}\right)+\varepsilon^{\beta} \rho^{\beta_{j}}\left(\widehat{\boldsymbol{T}}_{\alpha}^{\beta_{j}}+\widehat{e}_{\alpha}^{\beta_{j}} \boldsymbol{v}^{\beta_{j}}\right)=0 \quad j=1, \ldots, N,
$$

which, if written in terms of its microscale counterparts, corresponds directly with the jump condition across a discontinuous interface and is (Eringen and Maugin 1990):

$$
\boldsymbol{n} \cdot\left[\left[\rho \boldsymbol{v}(\boldsymbol{v}-\boldsymbol{w})-\underline{\underline{\boldsymbol{t}}}-\underline{\underline{t}}_{E}\right]\right]=0
$$

where $w$ is the velocity of the discontinuity. An alternate way of writing Eq. (32) for a single-phase fluid is in terms of the Maxwell stress tensor, $\underline{t}_{E}$, as defined by Eq. (10) where

$$
q_{e} \boldsymbol{E}+\boldsymbol{P} \cdot \nabla \boldsymbol{E}=\nabla \cdot \underline{\underline{t}}_{E}=\nabla \cdot\left(\boldsymbol{D} \boldsymbol{E}-\frac{1}{2} \varepsilon_{0} \boldsymbol{E} \cdot \boldsymbol{E} \boldsymbol{I}\right) .
$$

We note that the Maxwell stress tensor is symmetric if the polarization density, $\boldsymbol{P}$, is zero, and is typically used because it represents the interaction forces due to electric charges and fields, just as the mechanical stress tensor represents the interactions due to mechanical bonds.

Unfortunately, developing a corresponding Maxwell stress tensor for a multicomponent phase is not simple because in the derivation of Eq. (35), Faraday's law and Gauss' law are used, which in this formulation incorporates sources due to the presence of other phases, and also because symmetry is lost due to the two electric fields, the total electric field and the electric field generated by the species in phase $\alpha$. One could begin with the conservation of linear momentum in terms of Maxwell's stress tensor and upscale to get a corresponding Maxwell stress tensor with different upscaled definitions, but it is thought by some, e.g., Eringen and Maugin (1990), that the balance of linear momentum in terms of the Lorentz and Kelvin forces is more fundamental.

At this point, we have a full set of field equations (balance laws, Maxwell's equations) that hold at the macroscale. The material is viewed as overlaying continua with each component of each phase defined at each point in space and time. We next consider obtaining constitutive relations using hybrid mixture theory. 


\subsection{Constitutive Relations via Hybrid Mixture Theory}

To develop a full set of coupled equations using hybrid mixture theory, we use the second law of thermodynamics (Bennethum and Cushman 2002b), to develop macroscopic constitutive equations. The key assumption involves assuming a set of independent variables upon which all constitutive variables depend upon, and then using that assumption the entropy inequality is exploited in the sense of Coleman and Noll (1963).

Restricting ourselves to the conservation of mass and linear momentum, we have the following unknowns:

$$
\varepsilon^{\alpha}, \rho^{\alpha}, \boldsymbol{v}^{\alpha}, \boldsymbol{E}^{\alpha}, q_{e}^{\alpha}, \widehat{e}_{s}^{l}, \underline{t}^{\alpha}, \boldsymbol{P}^{\alpha}, \widehat{\boldsymbol{T}}_{\beta}^{\alpha} .
$$

The first four variables listed in (36) are primary unknowns. The last four variables are constitutive variables for which constitutive equations are needed in order to have the same number of equations as unknowns. For brevity, we assume a simple set of independent variables upon which all constitutive variables (not just the ones listed above, but also the variables appearing in the upscaled Maxwell equations and conservation of energy and entropy equation) must depend upon:

$$
\varepsilon^{l}, \rho^{\alpha_{j}}, \boldsymbol{v}^{l, s}, \boldsymbol{v}^{\alpha_{j}, \alpha}, \boldsymbol{E}_{\mathrm{T}}, \nabla \varepsilon^{l}, \nabla \rho^{\alpha_{j}}, \boldsymbol{d}^{l}, \nabla \boldsymbol{v}^{l_{j}, l}, \nabla \boldsymbol{E}_{\mathrm{T}},
$$

where $\alpha=l, s$ for the liquid or solid phase, respectively, $j=1, \ldots, N$ denotes the components within each phase (some of which are charged), a comma in the superscript denotes difference (e.g., $\left.\boldsymbol{v}^{l, s}=\boldsymbol{v}^{l}-\boldsymbol{v}^{s}\right)$, and $\boldsymbol{d}^{l}=\frac{1}{2}\left(\nabla \boldsymbol{v}^{l}+\left(\nabla \boldsymbol{v}^{l}\right)^{T}\right)$ is the rate of deformation tensor. Here we have assumed a porous material consisting of two phases, that is only deformable due to volumetric changes (not shear). If the porous media were to shear, it would be necessary to include strain in the list of independent variables.

Exploiting the entropy inequality to obtain constitutive equations for $\widehat{e}_{s}^{l}, \underline{\boldsymbol{t}}^{\alpha}, \boldsymbol{P}^{\alpha}, \widehat{\boldsymbol{T}}_{\beta}^{\alpha}$, substituting the results into the conservation of mass and conservation of linear momentum, neglecting the inertial terms and viscosity, and enforcing charge neutrality using a Lagrange multiplier, $\Lambda$ yields the generalized form of Darcy's law and Fick's law.

The generalized form of Darcy's law that results is (Bennethum and Cushman 2002b):

$$
\begin{aligned}
\boldsymbol{R} \cdot \boldsymbol{v}^{l, s}= & -\varepsilon^{l} \nabla p^{l}+\varepsilon^{l} \rho^{l}\left(\boldsymbol{g}+\boldsymbol{g}_{I}^{l}\right)-\pi^{l} \nabla \varepsilon^{l}+\varepsilon^{l} q_{e}^{l} \boldsymbol{E}_{\mathrm{T}}+\varepsilon^{l} q_{e}^{l} \nabla \Lambda \\
& +\varepsilon^{l} \boldsymbol{P}^{l} \boldsymbol{E}_{\mathrm{T}}-\sum_{j=1}^{N} \boldsymbol{r}^{l_{j}} \cdot \boldsymbol{v}^{l_{j}, l} .
\end{aligned}
$$

The coefficient $\boldsymbol{R}$ is the inverse of the conductivity matrix (up to porosity) and results from linearizing the constitutive expression for the exchange rate of momentum term, $\widehat{\boldsymbol{T}}_{s}^{l}$. The coefficient, $\boldsymbol{R}$, may be a function of all independent variables that are not necessarily zero at equilibrium (e.g., density, temperature, porosity, concentrations, etc). The first two terms on the right-hand side give the traditional Darcy's law, with the porosity typically absorbed into the conductivity matrix. The term involving $g_{I}^{l}$ implies that the effective "gravitational" term may include fluctuations in the electric field and its gradient (see the definition of $\boldsymbol{g}_{I}^{l}$ ). The next term, $\pi^{l} \nabla \varepsilon^{l}$, occurs when there is a strong interaction between the liquid and solid phase. The term $\pi^{l}$ (defined using the sign convention of Bennethum and Weinstein (2004)) measures the change in the energy of the liquid energy due to its proximity to the solid and is non-negligible in, e.g., swelling clays, swelling polymers, etc., and is a macroscopic term measuring what is typically called the disjoining pressure or osmotic pressure and indicates that the fluid will move from high porosity to low porosity with no other driving forces, 
such as a gradient in liquid pressure, present. See Bennethum and Weinstein (2004) for more details. The fourth term on the right-hand side is the upscaled Lorentz force. The following term, $\varepsilon^{l} q_{e}^{l} \nabla \Lambda$, states that the fluid will move so that charge neutrality (of the system) is enforced. If one does not assume charge neutrality exists in a system (for example, when an external electric field is applied), this term should be neglected. The next term is the upscaled Kelvin force, and the last term involving summation over species is a cross-effect term that captures the hydrating effect of ions. Because water has a polar moment, it naturally absorbs to ions (e.g., the hydrogen molecule to anions), causing a net bulk water movement in the direction of ions/cations. If species $l_{j}$ is not charged, then the corresponding $\boldsymbol{r}^{l_{j}}$ is zero, as there is (usually) no strong adsorption of water to non-charged species. It should be noted that one usually incorporates an electric field, $\boldsymbol{E}_{\mathrm{T}}$, or charge neutrality, but not both.

Of course, all of the terms on the right-hand side of Eq. (38) interplay-one could have a situation where the force due to the pressure potential is exactly counterbalanced by the Lorentz force resulting in no flow. As a specific example of this coupling, there is what is called the streaming potential (Newman and Thomas-Alyea 2004), which is usually described as an electric potential that arises in a charge-neutral system with a charged solid phase that undergoes deformation. The deformation of the solid phase causes electroneutrality to be violated, resulting in the movement of the charged particles in accordance with the resulting electric field. To observe this effect in Eq. (38), let us consider charge neutrality (keep the Lagrange multiplier), with negligible electric field (neglect $\boldsymbol{g}_{I}^{l}$ and the Kelvin and Lorentz forces), polarization, and swelling potential. Apply a pressure gradient. The liquid would move in bulk, causing the solid phase to deform, and the charge neutrality enforced between the liquid and solid phases would be violated, causing a new potential, $\Lambda$, that produces an electric field. See, e.g., Newman and Thomas-Alyea (2004) for an explicit example of such a calculation for charged particles in a fluid filled capillary tube at the microscale.

The generalized Fick's law provides the equation governing diffusion. With the assumptions incorporated here, we have

$$
\begin{aligned}
\sum_{k=1}^{N} \boldsymbol{r}^{l_{j k}} \cdot \boldsymbol{v}^{l_{k}, l}= & -\varepsilon^{l} \rho^{l_{j}} \nabla \mu^{l_{j}}+\varepsilon^{l} \rho^{l_{j}}\left(\boldsymbol{g}-\boldsymbol{g}_{I}^{l_{j}}\right)+\varepsilon^{l} q_{e}^{l_{j}} \nabla \Lambda \\
& +\varepsilon^{l} \rho^{l_{j}} z^{l} \boldsymbol{E}_{\mathrm{T}}-\left(\boldsymbol{r}^{l_{j}}\right)^{T} \cdot \boldsymbol{v}^{l, s},
\end{aligned}
$$

where $\mu^{l_{j}}$ is the chemical potential (change in energy with respect to quantity). The first term on the right-hand side is a generalization of the gradient of concentration, which leads us back to the traditional Fick's law. However, here we have additional terms. The tensors $\boldsymbol{r}^{l_{j k}}$ are material parameters that account for the coupling between the flow of one species and another. The tensor $\boldsymbol{r}^{l_{j}}$ is a material tensor that appears also in the generalized Darcy's law and accounts for the interaction between species $j$ in the liquid phase and the bulk liquid phase. Again the macroscopic body force incorporates not just gravity but also fluctuations in the electric field and its gradient (see the definition of $\boldsymbol{g}_{I}^{l_{j}}$ ). The term $\varepsilon^{l} \rho^{l_{j}} z^{l} \boldsymbol{E}_{\mathrm{T}}$ is a pseudoLorentz force, as the term in front of $\boldsymbol{E}_{\mathrm{T}}$ is not quite the charge of species $l_{j}$, and is negligible if the bulk porous medium is charge neutral.

To obtain the equivalent convection-diffusion equation, we make the following assumptions: (1) the effect of other species on the diffusion of species $j$ is negligible, so that the material coefficient tensor, $\boldsymbol{r}^{l_{j k}}$, is the identity tensor, $\boldsymbol{I},(2)$ there is no external electric field, so that we only enforce electric neutrality and that $\Lambda=-\Psi$, the electric potential, (3) the gravitational effects are negligible, and (4) the coupling with the bulk-phase fluid is negligible: $\boldsymbol{r}^{l_{j}}=\mathbf{0}$. Finally, let $\boldsymbol{r}^{l_{j j}}$ (no sum on $j$ ) be rewritten as $\left(\varepsilon^{l}\right)^{2} \rho^{l_{j}}\left(\boldsymbol{D}^{j}\right)^{-1}$ (note that 
from linearization, $\boldsymbol{r}^{l_{j j}}$ is a function of all independent variables that are not necessarily 0 at equilibrium), where $\boldsymbol{D}^{j}$ is the diffusion tensor. This yields

$$
\varepsilon^{l} \rho^{l_{j}} \boldsymbol{v}^{l_{j}, l}=-\boldsymbol{D}^{j} \cdot\left[\rho^{l_{j}} \nabla \mu^{l_{j}}+q_{e}^{l_{j}} \nabla \Psi\right],
$$

which is a generalized Fick's law. The term, $q_{e}^{l_{j}} \nabla \Psi$, is sometimes combined with the chemical potential to produce the electrochemical potential and represents the energy it takes to insert a charged particle into a charged system from infinitely far away (Newman and Thomas-Alyea 2004). For single-phase systems, there are explicit expressions for this potential, just as there are for chemical potentials in systems with no charges (Newman and Thomas-Alyea 2004).

To obtain the convective-diffusion equation for charged porous media, we substitute the expression for diffusion from (40) into the continuity equation for species $j$ (24), neglect mass transfer between phases and chemical reactions, divide through by $\rho^{l}$, and we obtain the simplest form:

$$
\varepsilon^{l} \frac{\partial c^{l_{j}}}{\partial t}+\varepsilon^{l} \boldsymbol{v}^{l} \cdot \nabla c^{l_{j}}-\nabla \cdot\left\{\boldsymbol{D}^{j}\left[c^{l_{j}} \nabla \mu^{l_{j}}+z^{l_{j}} c^{l_{j}} \nabla \Psi\right]\right\}=0,
$$

which we will use to compare with the homogenization approach.

And finally, we provide the resulting constitutive equation for the rate of exchange of mass for component $j$, see Eq. (18), from this framework (Bennethum and Cushman 2002b):

$$
\rho^{l_{j}} \boldsymbol{K}^{l_{j}} \widehat{e}_{s}^{l_{j}}=\tilde{\mu}^{s_{j}}-\tilde{\mu}^{l_{j}}+\rho^{l_{j}} z^{s_{j}} G^{l_{j}} \widehat{Z}_{s}^{l_{j}},
$$

where $\boldsymbol{K}^{l_{j}}$ and $G^{l_{j}}$ are linearization coefficients that must be measured, and $\widetilde{\mu}^{l_{j}}$ and $\tilde{\mu}^{s_{j}}$ are the electrochemical potentials for component $j$ in the liquid and solid phases, respectively. For non-charged systems, the electrochemical potentials reduce to the chemical potentials and the rate of exchange of charge, $\widehat{Z}_{s}^{l_{j}}$, is zero. Thus we see that the larger the difference in the electrochemical potentials, the faster phase transition occurs.

We note that because these equations are developed at the macroscale by exploiting the entropy inequality, the resulting equations satisfy the Onsager's reciprocity relations (Onsager 1931a,b), which, in its simplest terms, states that the matrix relating the forces and fluxes of any system near equilibrium must be symmetric. One can see this relationship in, for example, the appearance of the coefficient $\boldsymbol{r}^{l_{j}}$ in both Darcy's equation (38) and Fick's equation (39), coupling diffusion with bulk motion. However, this formulation does not take advantage of any knowledge of constitutive equations that are known at the microscale (such as the Nernst-Planck equation (15)), nor the microscale geometry, other than what is captured by the volume fraction. The latter restriction can be somewhat alleviated by including additional parameters in the set of independent variables, such as the liquid-solid interfacial area density (area per representative volume of the porous media). See for example (Hassanizadeh and Gray 1990). One consequence of this formulation is that the system of equations requires that all coefficients in the constitutive equations are directly formulated at the macroscale, requiring that they be measured via macroscopic experiments.

\subsection{Homogenization}

An alternative approach is to upscale both field equations and constitutive equations from the microscale to the macroscale. Two such approaches are volume averaging in the sense of Whitaker (e.g., Rio and Whitaker (2000)) and homogenization (Schmuck and Bazant 2015). Both approaches rely on having sufficient information at the microscale in order to obtain 
macroscopically feasible results as well as physical intuition in order to close the system of equations (for volume averaging), or for determining powers in the expansion coefficients (homogenization), but this is common for all upscaling approaches (HMT requires a selection of independent variables and intuition to determine the coupling and degree of Taylor series expansion). Here we focus on homogenization and begin with probably the simplest formulation for charged porous media as given by Schmuck and Bazant (2015).

At the microscale, we begin with the Poisson-Nernst-Planck equations for ion transport in a fluid, in Eqs. (6) and (15), assume two charged species in the fluid phase, a cation and an anion, and a periodic structure consisting of spherical solids that have a surface charge density. A periodic structure consists of spherical solids with a solid phase that has a surface charge density. Following the classical homogenization procedure (not trivial as once the solid phase is charged the system is nonlinear), the governing equations at the macroscale are given as (notation modified to be consistent with that which is used here) (Schmuck and Bazant 2015):

$$
\begin{aligned}
& \varepsilon^{l} \frac{\partial c^{l_{j}}}{\partial t}=\nabla \cdot\left(\boldsymbol{D}(\boldsymbol{v}) \cdot \nabla c_{0}+z^{l_{j}} c^{l_{j}} M \nabla \Psi\right) \\
& -\nabla\left(\epsilon_{0} \nabla \Psi\right)=\left(c^{l_{+}}-c^{l_{-}}\right)+\rho_{s}
\end{aligned}
$$

where three charged species are considered, cations and anions, with mass concentration $c^{l_{+}}$and $c^{l_{-}}$, respectively, and a solid surface, with charge density, $\rho_{s}$. The first equation is the generalized convection-diffusion equation, and the second is a macroscopic version of Gauss' equation, which together could be referred to as macroscopic Poisson-Nernst-Planck equations. The nonlinearity of the Poisson-Nernst-Planck equations makes it challenging to upscale, and consequently, the convection-diffusion equation is missing the convection term explicitly, but is incorporated through a transformation of the dependent variable, e.g., $c^{ \pm}=c^{ \pm}\left(\boldsymbol{x}-\frac{t \boldsymbol{v}^{*}}{r}, t\right)$, where $\boldsymbol{v}^{*}$ is a "suitably averaged fluid velocity" (Schmuck and Bazant 2015). Note that the diffusion coefficient is now a tensor (unlike at the microscale) and one can solve (usually numerically) a problem using the assumed microscopic geometry to determine exactly what $\boldsymbol{D}$ is, and determine how microscale changes will affect the macroscale diffusion tensor.

Although this provides probably the simplest version of a macroscale model obtained via homogenization, this particular formulation has a few drawbacks. Besides the missing convective term (again, incorporated in the independent variables), this formulation does not incorporate the conservation of momenta for the solid (and thus does not have an equation that determines the change in porosity), nor for the fluid (so there is no, e.g., streaming potential). Consequently, the resulting macroscopic equations do not satisfy the Onsager reciprocity, which the authors readily admit. Further, the placement of the anions/cations at the microscale is not taken into account (via, e.g., the double-layer theory), so that this information is not accounted for in the macroscopic diffusion tensor.

An example of a homogenization approach that alleviates many of these shortcomings is that of Moyne and Murad (2006). In this work, the microscale equations include the Poisson problem (Gauss' equation), Stokes problem with the Lorentz force, the constitutive equation for the fluid stress tensor incorporating the Maxwell stress tensor, the PoissonNernst convective-diffusion equation using the electrochemical potential (Poisson-NernstPlanck equation), the equilibrium form of the conservation of momentum for the solid phase assuming the stress tensor is linearly related to the strain tensor, and the continuity equation for the solid phase charges assuming that the fluxes of the charged particles is due only to the displacement of the solid. Because Moyne and Murad (2006) is interested in modeling 
montmorillonite clay, the geometry of the periodic structure is taken to be two parallel solid plates with a fluid consisting of water with two charged species, an anion and a cation. To help deal with the highly variable-dependent variables liquid pressure, liquid ion concentrations, and liquid electric potential due to charge distribution (decomposed into that which arises from the electric double layer and that which arises from the streaming potential), the authors make a clever change of variables to concentration of the cation or anion, $c_{b}$, pressure, $p_{b}$, and electric potential (due to streaming potential only), $\Psi_{b}$, for a fictitious bulk fluid that is in equilibrium with the fluid between the charged platelets. Because the fictitious fluid is not in contact with the charged solid phase, it is electrically neutral so that the concentration of anions and cations are equal. Assuming a microscale velocity in the $x$-direction (parallel to the plates), the first approximation at the macroscale of the generalized Darcy's (Moyne and Murad 2006, Equation (4.50)) and convection-diffusion equation (Moyne and Murad 2006, Equation (4.53)) is:

$$
\begin{aligned}
& \boldsymbol{v}^{l, s}=-\boldsymbol{K}_{P} \nabla_{x} p_{b}-\boldsymbol{K} \cdot \nabla_{x} c_{b}-\boldsymbol{K}_{E} \cdot \nabla_{x} \Psi_{b} \\
& \frac{\partial}{\partial t}\left(\varepsilon^{l} G_{ \pm} c_{b}\right) \\
& \quad+\nabla \cdot\left[G_{ \pm} c_{b} \boldsymbol{v}_{ \pm}-\varepsilon^{l}\left(\boldsymbol{D}_{ \pm} \cdot \nabla_{x} c_{b} \pm \boldsymbol{D}_{ \pm}^{e} c_{b} \nabla \Psi_{b}+\boldsymbol{D}_{ \pm}^{p} \cdot \nabla_{x} p_{b}\right)\right]=0
\end{aligned}
$$

where subscript $b$ refers to the quantity in the bulk phase that is in equilibrium with the liquid phase, and $G$ relates the concentration of the bulk component to the electric field generated by the double-layer theory using the Poisson-Boltzmann equation at the microscale. We again note that each coefficient can be obtained explicitly by solving a problem at the microscale. It can be shown that the symmetry of Onsager's relations holds if it is assumed that the electrochemical potentials of the ions do not fluctuate in the micropores (Moyne and Murad 2006).

We note that even though the hybrid mixture theory, HMT, and homogenization approaches are quite different approaches, the resulting macroscopic equations have quite a bit in common. To compare the generalized Darcy's law of HMT, Eq. (38) with that of homogenization, Eq. (43), we rewrite Eq. (38) using the following relations

$$
\begin{aligned}
G^{l} & =\psi^{l}+\frac{p^{l}}{\rho^{l}}=\sum_{j=1}^{N} c^{l_{j}} \mu^{l_{j}} \quad G^{b}=\psi_{b}+\frac{p_{b}}{\rho_{b}}=\sum_{j=1}^{N} c^{b_{j}} \mu^{b_{j}} \\
\mu^{l_{j}} & =\frac{\partial\left(\rho^{l} \psi^{l}\right)}{\partial \rho^{l_{j}}}=\mu^{b_{j}}=\frac{\left(\rho_{b} \psi_{b}\right)}{\partial \rho^{b_{j}}} \\
p^{l} & =\sum_{j=1}^{N} \rho^{l} \rho^{l_{j}} \frac{\partial \psi^{l}}{\partial \rho^{l_{j}}} \quad p_{b}=\sum_{j=1}^{N} \rho_{b} \rho^{b_{j}} \frac{\partial \psi_{b}}{\partial \rho^{b_{j}}} \\
\pi^{l} & =\varepsilon^{l} \rho^{l} \frac{\partial \psi^{l}}{\partial \varepsilon^{l}} \\
\psi^{l} & =\psi^{l}\left(\varepsilon^{l}, \rho^{l_{j}}\right) \quad \psi_{b}=\psi_{b}\left(\rho^{b_{j}}, p_{b}\right),
\end{aligned}
$$

where $G$ is the Gibbs potential (energy per unit mass), $\psi$ is the Helmholtz potential (energy per unit mass), $\rho^{l_{j}}=c^{l_{j}} \rho^{l}$, and $\mu^{l_{j}}$ is the chemical potential for the $j$ th component in the liquid phase. The equality of the chemical potentials between the liquid phase and the bulk fluids comes from the definition of thermodynamic equilibrium of the pseudo bulk phase. 
Using these relations results in an equivalent form of the HMT form of Darcy's law

$$
\begin{aligned}
\boldsymbol{R} \cdot \boldsymbol{v}^{l, s}= & -\varepsilon^{l} \nabla p^{l}+\varepsilon^{l} \rho^{l}\left(\boldsymbol{g}+\boldsymbol{g}_{I}^{l}\right)-\pi^{l} \nabla \varepsilon^{l}+\varepsilon^{l} q_{e}^{l} \boldsymbol{E}_{\mathrm{T}}+\cdots \\
= & -\varepsilon^{l} \rho^{l} \nabla G^{l}+\varepsilon^{l} \rho^{l} \mu^{l_{j}} \nabla c^{l_{j}}+\varepsilon^{l} q_{e}^{l} \boldsymbol{E}_{\mathrm{T}}+\cdots \\
= & -\varepsilon^{l} \rho^{l} c^{l_{j}} \nabla \mu^{l_{j}}+\varepsilon^{l} q_{e}^{l} \boldsymbol{E}_{\mathrm{T}}+\cdots \\
= & -\varepsilon^{l} \rho^{l}\left[\sum_{j} c^{l_{j}} \frac{\partial \mu^{b_{j}}}{\partial p_{b}}\right] \nabla p_{b}-\varepsilon^{l} \rho^{l} \sum_{j}\left[c^{l_{j}} \frac{\partial \mu^{b_{j}}}{\partial c^{b_{j}}} \nabla c^{b_{j}}\right] \\
& +\varepsilon^{l} q_{e}^{l} \boldsymbol{E}_{\mathrm{T}}+\cdots
\end{aligned}
$$

which is the same form as that obtained via homogenization (43).

Comparing the convection-diffusion equations among the three approaches requires doing a similar manipulation for (41). Assuming that the chemical potential of the liquid- and bulkphase fluids is equal, $\mu^{l_{j}}=\mu^{b_{j}}$, and that the Helmholtz potential and hence the liquid chemical potential of the bulk fluid are functions of the bulk pressure and concentrations, $\mu^{b_{j}}=\mu^{b_{j}}\left(p_{b}, c^{b_{j}}\right)$ yields

$$
\begin{aligned}
& \varepsilon^{l} \frac{\partial c^{l_{j}}}{\partial t}+\varepsilon^{l} \boldsymbol{v}^{l} \cdot \nabla c^{l_{j}} \\
& -\nabla \cdot\left\{\boldsymbol{D}^{j} \cdot\left[\sum_{i} c^{l_{j}} \frac{\partial \mu^{b_{j}}}{\partial c^{b_{i}}} \nabla c^{b_{i}}+c^{l_{j}} \frac{\partial \mu^{b_{j}}}{\partial p_{b}} \nabla p_{b}+z^{l_{j}} c^{l_{j}} \nabla \Psi\right]\right\}
\end{aligned}
$$

and we readily see the comparison between the three formulations are fairly close, up to the convective term and time derivative terms.

It should be noted that these works are far from complete and are undergoing evolutions. For example, in homogenization, work incorporating three scales to, e.g., model montmorillonite clay incorporating ion-ion correlation effects (Le et al. 2015), and even size exclusion effects are exciting new developments.

\section{Charged Porous Media in Real-World Applications}

There is a wide range of applications, in which utilization of the charge property controls the success of the application. For brevity, we introduce only a few examples with physical scales spanning several orders of magnitude from centimeter to kilometer. The provided examples cover use of clays to make impermeable layers, utilizing the charged surface of rocks for enhanced oil recovery, charged media in electrochemical batteries, and drug delivery.

\subsection{Clays}

The terminology clay refers to soils with particle sizes finer than $2 \mu \mathrm{m}$ in geo-engineering applications, and the term clay minerals refer to secondary minerals that are the products of chemical weathering of primary minerals of metamorphic and sedimentary rocks (Yong et al. 2010). At the molecular scale, the clay minerals consist of two basic crystal units of silicon oxygen tetrahedral unit and aluminum/magnesium/iron-oxygen-hydroxyl octahedral unit as shown in Fig. 2. The crystal units of clay minerals carry an unbalanced electrical charge on the surfaces and edges. Possible sources of the unbalanced electrical charge are related to: (i) the positively charged ions that are generally located in the space between 


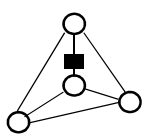

Tetrahedral unit

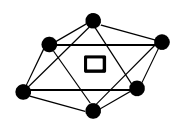

Octahedral unit

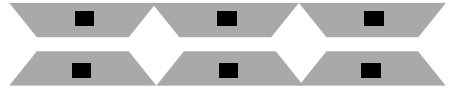

Tetrahedral sheet

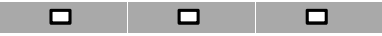

Octahedral sheet

\begin{tabular}{|llllll}
\hline Oxygen $\quad$ Si $\quad \bullet \quad$ Hydroxyl $\square$ Al, Mg, Fe \\
\hline
\end{tabular}

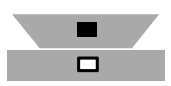

Basic unit 1:1 cell

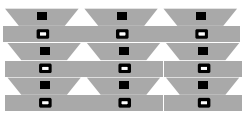

Kaolinite

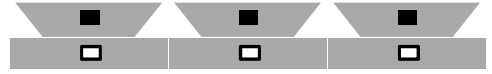

Basic unit layer

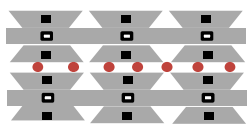

Illite

- Potassium ions

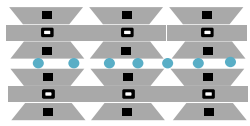

Smectite

Discrete layers of $\mathrm{H}_{2} \mathrm{O}$ and exchangeable cations

Fig. 2 Basic microstructure units of clay mineral. Adopted and reproduced from Yong et al. (2012)

the basic unit layers, whereas the oxygen or hydroxyl ions on the surface of the unit layer are negatively charged, (ii) the partial replacement of $\mathrm{Si}^{4+}$ by $\mathrm{Al}^{3+}$ in the tetrahedral unit or of $\mathrm{Al}^{3+}$ by $\mathrm{Mg}^{2+}$ in the octahedral unit that results in a net negative charge on the unit layers (isomorphous substitution, e.g., in montmorillonite), and (iii) the edges of unit layers where the tetrahedral silica sheets and octahedral sheets are disrupted, and primary bonds are broken. Charge imbalance resulting from the isomorphous substitution is balanced by the exchangeable cations that are located between the unit layers and on the surfaces of particles. The arrangement of unit layers for three common clay minerals including kaolinite, illite, and smectite is shown in Fig. 2.

\subsubsection{Clays in Geo-environmental Barriers}

Clays play an important role in developing engineering solutions for geo-environmental problems. In particular, clays with a large percentage of smectite clay minerals are of great interest in geo-environmental applications. An example of such engineering utilization of clays is the widespread application of bentonite clay (which has a high percentage of smectite in its composition) as engineered barriers for waste disposal and containment systems. Bentonite has a high capacity of water adsorption and swelling characteristics alongside its highly charged surfaces (average surface area of sodium bentonite is $700-800 \mathrm{~m}^{2} / \mathrm{g}$ ). It has a high cation exchange capacity, originating mainly from the negatively charged surfaces 
of bentonite. Bentonite is used as part of the composite systems of geosynthetic clay liners (GCL) at the bottom and top of waste disposal/landfill sites. GCL comprises a layer of partially hydrated bentonite sandwiched between two layers of geomembranes and/or geotextiles. GCL can provide a low permeability hydraulic barrier to minimize the infiltration of hazardous chemicals into the subsurface soil and groundwater below the landfill (landfill liner system) or prevent runoff infiltration into the waste deposition (landfill cap system). In high-level nuclear waste (HLW) disposal sites, compacted bentonite is a component in vertical barriers in order to isolate the contaminated sites and prevent migration of groundwater through the contaminated sites. Compacted bentonite not only provides a mechanically stable environment and minimizes the percolation of groundwater, but it also has a high swelling pressure, low diffusion coefficient, and high attenuation capacity, which are mainly related to the highly charged smectite surfaces.

\subsubsection{Diffusion in Clays}

The pore system of clays is a distribution of pore sizes in which mass transport is heavily affected by the surface interactions and charge imbalance. At least three porosity scales can be identified (Pusch et al. 2007; Sedighi and Thomas 2014) (see Fig. 3): (i) the interlayer or microporosity that corresponds to the pores associated with interlayer space between the unit layers (generally $<2 \mathrm{~nm}$ ), (ii) mesoscale pores that corresponds to space existing between the particles $(2-50 \mathrm{~nm})$, and (iii) the macroscopic porosity corresponding to the inter-aggregate voids $(>50 \mathrm{~nm})$. The interlayer pores only contain water and exchangeable ions. Ions form a diffuse double-layer system around the particles that normally exist to balance the charge of the clay surface. The diffuse double layer that is formed at the meso- and macroscale contains both cations and anions as shown in Fig. 1b (Bradbury and Baeyens 2003; Bourg et al. 2003; Wersin et al. 2004). The vicinal fluid consists of the fluid within the interlayer and a small portion of water in the micropores that is close to the particle surface and is typically considered relatively immobile compared to water in the macropores, and some authors treat this portion of water as part of the solid phase (Pusch 1994; Hueckel 1992). Practically, the fluid in the interlayer pores contributes very little to fluid migration, whereas the microand macropores are likely to act as major fluid pathways (Pusch 1994; Hueckel 1992). The diffusion of charged species in compacted bentonites compared to diffusion in free water is more strongly influenced by geometrical factors caused by the complex microstructure of the smectite clay (Leroy et al. 2006). In addition, gas transport in smectite is a highly reactive process that involves interaction between water and clay minerals and chemicals (Sedighi et al. 2015). Experimental investigations have been conducted to understand the diffusion of ionic species in smectite, demonstrating that the diffusion rate and the effective diffusion coefficients of neutral species, anions, and cations vary considerably with the type of chemical species in water resident in compacted bentonite and the imbalanced charged nature of particles (Muurinen et al. 2007; Kozaki et al. 2005; García-Gutiérrez et al. 2004; Van Loon et al. 2007; Glaus et al. 2007). The theoretical understanding of the effective mechanisms of the diffusion of ions in compacted bentonite has also been advanced by Lehikoinen et al. (1995) and Leroy et al. (2006). García-Gutiérrez et al. (2004) studied the diffusion properties of a smectite-rich clay to a chloride tracer compared with the tritiated water that showed all the meso- and macropores in compacted smectite studied are available for diffusion of neutral species. On the other hand, the accessible porosity for a chloride tracer was found to be only $2-3 \%$ of the total porosity, demonstrating a significant anionic exclusion. The increased diffusion of cations has also been observed and explained by the interlayer diffusion (Bourg et al. 2003; Glaus et al. 2007) or by surface diffusion in the diffuse double 

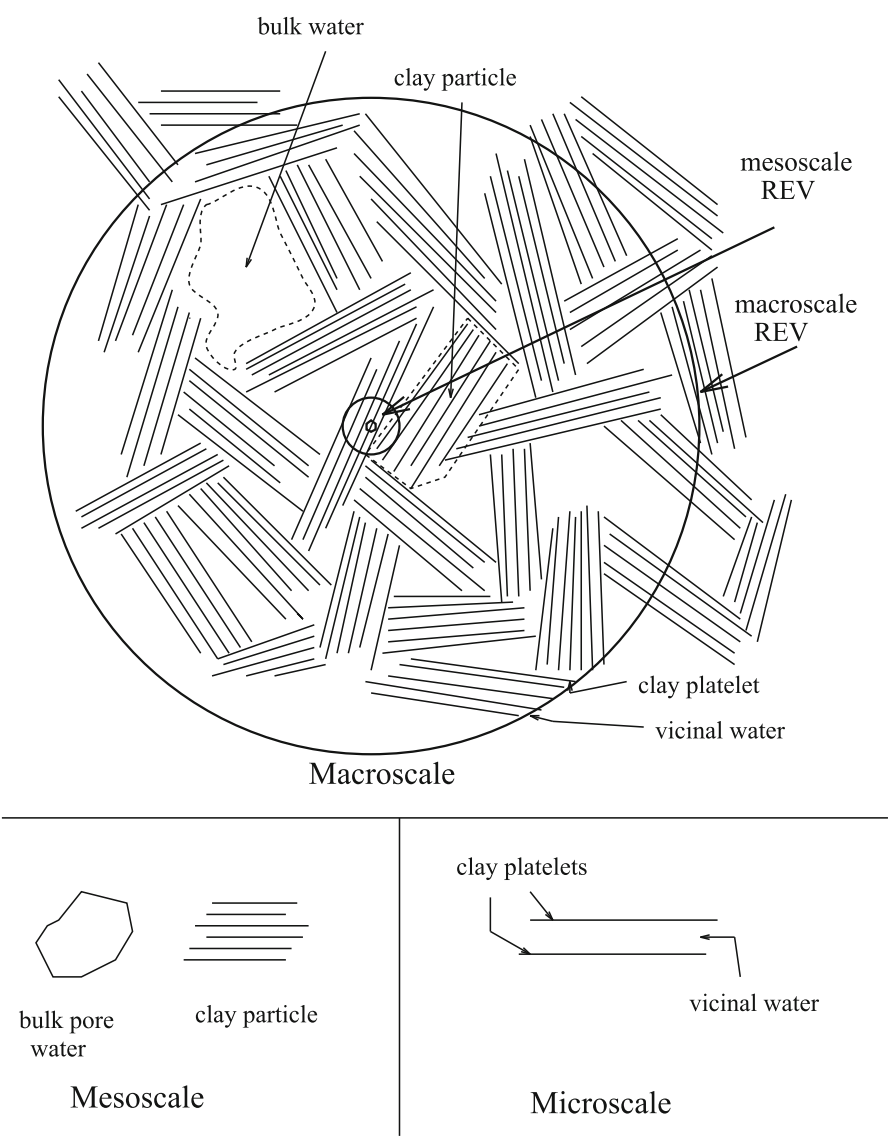

Fig. 3 A schematic presentation of the multiscale structure of the montmorillonite clay

layer (DDL) (Leroy et al. 2006; Appelo et al. 2010). The above-mentioned findings indicate that in general larger values for the effective diffusion coefficients of cations and smaller values for anions than those for water tracers (neutral species) have been found commonly in compacted clays (Appelo and Wersin 2007). The results of investigations of the diffusion of anions and cations suggest that accessible porosity and geometrical factor are likely to be different for various tracers in compacted bentonite that are key to accurate reactive transport modeling and prediction of processes in swelling clays (Sedighi et al. 2018).

\subsection{Role of Charged Surfaces in Low-Salinity Waterflooding}

Enhanced oil recovery refers to a group of chemically enhanced technologies to extract oil from the reservoirs. Electrokinetic effects play the central role in several enhanced oil recovery methods including foam flooding (Farajzadeh et al. 2012) and low-salinity waterflooding (Mahani et al. 2015; Joekar-Niasar and Mahani 2016; Aziz et al. 2018).

In low-salinity waterflooding, water with an engineered composition is injected to produce more oil compared to conventional waterflooding. Several hypotheses have been proposed to be responsible for the extra oil recovery, among which wettability alteration due to the 
expansion of the electrical double layer in clay-rich sandstones (Ligthelm et al. 2009) and ion exchange between the fluids and clays/sandstone rock (Lager et al. 2006) are the two most likely mechanisms. A detailed literature review on low-salinity waterflooding can be found in Bartels et al. (2019).

The double-layer expansion mechanism deals with the thickness and shape of the water film (Choi et al. 2010; Basu and Sharma 1996; Dimitrova et al. 2001; Binks et al. 1997; Manica et al. 2007; Onsager 1949). Recently, in a theoretical study it has been shown that the pressure induced by the electrokinetic and osmotic effects are highly nonlinear and depend on the film thickness. In thin films exposed to a lower ionic strength, the electrokinetically induced pressures lead to the increase in disjoining pressure and consequently change of contact angle toward the more water-wet state (Churaev and Sobolev 1995; Joekar-Niasar and Mahani 2016).

The multivalance ion exchange deals with the interfaces between the fluids and rocks. Several studies have shown that there is a distinct difference between the behavior of monovalent and divalent ions, as divalent ions tend to become sorbed (chemically attached to the substrate) (Nicolini et al. 2017; Sohrabi et al. 2017) and can potentially lead to the inversion of the surface charge, which changes the surface forces from attractive to repulsive conditions. In these two mechanisms, the surface electrochemistry and electrokinetic forces are the key factors that can explain the microscopic phenomena during the low-salinity waterflooding (Rivet et al. 2010). To simulate wettability alteration in porous materials, a complex multiscale sets of equations need to be solved. At the Darcy scale, the two-phase flow with variable wettability needs to be solved. However, at the microscale, the wettability alteration can be triggered as the interaction of several factors (e.g., surface chemical interactions, electrokinetic effects in the diffuse layer) lumped in the Maxwell stress tensor (Eq. 10). The complete physical phenomena at the microscale can be simulated by coupled Poisson-Nernst-Planck and flow equations. Currently, there is no comprehensive multiscale model that integrates electrokinetic effects, surface chemistry, disjoining pressure, wettability alteration, and twophase flow in an integrated model.

\subsection{Electrochemical Models for Rechargeable Batteries}

Lithium-ion and other rechargeable batteries are another important example of charged porous media (Newman and Tiedemann 1975). Here, to obtain a higher power density, porous solid electrodes with a high surface-volume ratio are generally used. Increasing the surface available for the electrochemical reaction (ion intercalation/de-intercalation) between the solid electrodes and the liquid electrolyte is, in fact, one of the key design objectives, together with an efficient transport of ions in the electrolyte, through the porous electrode matrix and the separator (often porous itself). One of the main challenges is therefore to design the porous structure, for a given battery chemistry, to optimally balance these two mechanisms. While the transport models will be discussed at length in the following, it is important to highlight that the electrochemical reactions are highly nonlinear phenomena, usually described by the Butler-Volmer equation (Rubi and Kjelstrup 2003). This is an effective macroscopic description of the ion exchange at the electrode surface, relating the electrical current with the so-called over-potential, which is computed as the difference between the potential drop between electrolyte and electrode, and an equilibrium "open-circuit" potential. The latter is usually measured experimentally as a function of the ion concentration (state of charge) at equilibrium. 
A widely used model for lithium-ion batteries is the one originally developed in the seminal work of Newman and collaborators (Newman and Tiedemann 1975). This, despite the several assumptions and subsequent improvements, is still the basis for most of the recent simulation, optimization, and control studies. The peculiarity of this model is that, due to the large ratio between the diffusivity of ions in the solid electrodes and the one in the electrolyte (liquid) that induces a strong coupling between the scales, it is formulated in a hybrid macro-microapproach, also known as pseudo-two-dimensional (P2D) because originally developed for a mono-dimensional macroscopic space variable with a one additional microscopic dimension. This has been subsequently more generally derived by homogenization by several authors (Arunachalam et al. 2015; Richardson et al. 2012; Ciucci and Lai 2011).

The basic assumptions include electroneutrality, negligible fluid momentum, nondeformable pores, and the transport equations of only one species (ion concentration) in two phases (solid and liquid electrolyte). For the liquid electrolyte phase, we can refer to Eqs. (40-41), with appropriate chemical potential (see also Eq. 15), and effective macroscopic diffusivity, together with a macroscopic Poisson equation for the potential (with effective macroscopic conductivity). The source term in both these equations is proportional to the nonlinear Butler-Volmer equation that here represents the transfer of charges between the phases (and depends on ion concentration fields and potential in both phases). Although this equation is treated as a macroscopic balance (developed as a way to include surface DLVO effects and electrochemistry) for the local current at the electrode surface, its derivation does not usually incorporate any correction or upscaling for a porous medium. This assumption is appropriate, provided a slow reaction timescale compared to the electrolyte diffusion, and we can therefore approximate the total source term locally with the Butler-Volmer equation, simply scaled by the specific surface.

Unfortunately, these approximations are not valid for the solid phase in which the ion diffusivity is typically extremely slow (usually of at least four orders of magnitude). This is equivalent to the well-known problem of upscaling high-contrast media. Therefore, in principle, the full microscale solution would be needed at each point. However, assuming the solid phase is made of non-connected spherical particles, Newman and Tiedemann (1975) proposed a hybrid micro-macromodel where, at each macroscopic point, a diffusion equation is solved for the ion concentration in a microscopic radial coordinate only. Here, the phase exchange term (the Butler-Volmer kinetics) is applied as a flux at the external boundary. Several improvements (polydispersed particles) or further approximations (e.g., single-particle model) have been since proposed. The potential of the solid is instead averaged (or homogenized) over the solid electrode porous structure (partially in contradiction with the assumption of non-connected spherical particles) and represented as a macroscopic Poisson equation with effective solid conductivity.

While this model has clearly several limitations, it still represents a milestone in understanding the dynamic behavior of lithium-ion batteries, as a compromise between more complex electrochemical molecular models and system-scale equivalent circuit models for control and real-time applications.

\subsection{Drug Delivery}

There are multiple means of introducing drugs into the body, e.g., orally, across a membrane such as a nasal membrane, and adsorption through the skin. One commonly used mechanism is a hydrogel, which is typically composed of a swelling (hydrophilic) polymer that has the 
morphology of spaghetti (Feng et al. 2010). The hydrogel is carefully laced with the drug of interest (e.g., Aleve), and as the hydrogel comes into contact with body fluid, it swells, increasing the pore size, allowing the drug to diffuse out. The polymer can be cross-linked, which keeps the polymer from dispersing completely, or not. The physical process of the liquid entering into a hydrogel is due to osmotic pressure and is a consequence of the liquid having a preference to being next to the solid polymer instead of being in a bulk phase. An example of a more complicated drug delivery system is an osmotic-controlled release oral delivery system (Malaterre et al. 2009), which involves encapsulating a hydrogel in a rigid, semipermeable outer membrane that allows fluid to enter in, building up the osmotic pressure inside, which then pushes the drug out of the capsule through small laser holes that have been drilled into the outer membrane. In more recent years, electrical stimulation has been used to release a drug from an implanted osmotic-controlled release device resulting in a system that can administer a precise drug amount at a given time and location (Yi et al. 2015). At the microscale, these mechanisms are a consequence of the solid polymer having a slightly charged surface, resulting in hydration by water, or in the case of the on-demand release mechanism, an electric field that affects the deformation of the polymer. There are several mathematical modeling approaches (see Siepmann and Siepmann 2008 for a nice review), including hybrid mixture theory (Weinstein et al. 2008a, b).

\subsection{Biomechanics}

A human body is a structure where the electrical double layers are ubiquitous. While human tissue is mainly composed of water, the body appears without a doubt as a solid structure. The high water content is vital as renewal of tissue is constantly going on, and supply and drainage of material have to happen from capillaries to cells through diffusion. The reason why, despite the high water content, human tissues appears solid is because most of the water is bound in electrical double layers in and outside the living cells. The cytoplasm is understood as nanoporous network of biological polymers, named cytoskeleton. The cytoskeleton is charged in many living cells. The extracellular space of cartilage, intervertebral disk, and bone have fixed charges very much like clay minerals has. One of the most salient features of the remodeling process of a human tissue is that the tissue itself organizes its renewal so as to resist existing mechanical load better. The ability of biological tissue to sense its mechanical load is known as mechanotransduction. There is a suspicion that electrical double layers may play an important role in this mechanotransduction.

Large deformations usually play an important role in applications of biological porous media (Huyghe and Janssen 1997). The osmotic pressure in blood is tightly regulated by the kidneys, indicating that osmotic pressure is a vital aspect of physiology. Small departures from physiological salt concentrations quickly result in swelling and disease, and it was known even thousands of years ago (ayurvedic scriptures) that swelling was a key symptom of disease. The osmotic pressure defined in Eq. (12) is the change in chemical potential of the water because of the vicinity of ions. The change of chemical potential drives osmosis, ensuring that the majority of water molecules in the human body are bound to the (ionized) solid. Differences in charge density between the intracellular and extracellular environment cause differences between intracellular osmotic pressure and extracellular osmotic pressure. This difference in osmotic pressure across a permeable cell membrane results in a jump in mechanical pressure across the membrane. The smooth round shape of cell membranes is a direct consequence of this pressure jump. Ions are sensitive to the difference in charge density as well. Intracellular ionic concentrations differ significantly between intracellular space and 
extracellular space (Guyton and Hall 1996). Part of these differences can be explained by the hybrid mixture theory mentioned in the previous sections. A key feature of cell physiology is the jump in electric potential across the cell membranes. This jump amounts to several tens of $\mathrm{mV}$. Equating the electrochemical potential, (Eq. 13), of the intracellular space to the electrochemical potential of the extracellular space results in quantitative predictions of this voltage jump. Nevertheless, some caveat should be mentioned in the context of a multiple-ion system like a human cell. In most ionic theories describing electrical double layers, ions are described as point charges. This implicitly means that sodium ion with charge +1 is identical to a potassium ion with charge +1 , while size of the $\mathrm{K}^{+}$is 152 picometer and size of $\mathrm{Na}^{+}$is 116 picometer and thus cannot be treated identically. More advanced theories are needed to deal with heteroionic systems and the interaction between ions of same charge. Substantial effort has been invested in the understanding of why potassium concentration inside cells is higher than outside, and why sodium concentration inside cells is lower than outside. Such a dichotomy cannot be explained by most existing ionic theories. Once deformations come into the picture, the electrochemical potential $\mu_{i}$ in Eq. (13) depends not only on electric potential and concentration, but also on strain (Huyghe and Janssen 1997; Huyghe et al. 2009). This dependence is the pivotal property allowing the electrical double layers to sense strain. As strain changes, electrochemical potential changes and by virtue of Fick's law ions are secreted or absorbed, meaning that the mechanical signal of strain is translated into an electrical one. In endothelial cell physiology, it has been demonstrated that an ionic species nitric oxide $\mathrm{NO}^{-}$is secreted according to the strain to which the endothelial cell is submitted (Ignarro 2000). Although it is too simplistic to present the response of a complex cell such as the endothelial cell as a purely electrochemical response, the very principle of mechanotransduction through ionic flux is demonstrated by this example.

\section{Limitations, Challenges, Perspective}

In this paper, the fundamental concepts used to model flow and transport in charged porous media have been introduced. While the microscopic description of physical and chemical processes in charged porous media have been explained, Darcy-scale models are required to simulate the behavior of these systems at larger physical scales.

We first note that classical microscale equations (e.g., DLVO theory) are based on electrostatic forces and do not model non-equilibrium conditions, see Sect. 2. This means to solve the ions transport in electrically charged systems, the electric field needs to be coupled with the transport equation (referred to as Poisson-Nernst-Planck Eqs. (6), (15), and (16)). As a result, the expression for the disjoining pressure based on the equilibrium Poisson-Boltzmann relation is not valid. Similar to the preliminary work of Joekar-Niasar and Mahani (2016), non-equilibrium disjoining pressure and non-equilibrium osmotic pressure should have constitutive expressions that are valid at non-equilibrium.

It is important to note that there are several underlying assumptions in Poisson-Boltzmann and Poisson-Nernst-Planck equations that limit their validity under some conditions such as high concentrations. For example, in the original form of these theories, ions are considered as point charges (no size assigned) and consequently the concentration can theoretically increase to infinity. Although this assumption might be valid at low concentrations, at high concentrations the size of ions is important and leads to significant deviation of observations from theory. The impact of size of ions on electrokinetics, referred to as Steric effects, has been incorporated in Poisson-Boltzmann and Poisson-Nernst-Planck by several researchers 
such as Kilic et al. (2007), Gupta and Stone (2018), Kilic et al. (2006) and Kornyshev (2007). Inclusion of size of ions in the modeling can potentially impact the modeling of electrokinetics in enhanced oil recovery where the formation brine can have a molar concentration of larger than 1 (McGuire et al. 2005).

Electroneutrality is another important condition that should be carefully considered in computational simulation of electrokinetics in porous media. The condition applied to enforce electroneutrality is highly scale dependent. For example, electroneutrality should only be enforced within the electrical diffuse layer if the surface charge density is incorporated as well, while in the bulk concentration, where the electrical potential is negligible, the net charge concentration should be zero. Further, expressions for the cross-coupling diffusion coefficients are based on the bulk concentration assumption (Boudreau et al. 2004a), implying that the diffusion of co- and counterions in the electrical diffuse layer has been assumed negligible. While this assumption might be valid for porous media with large pores (compared to the Debye length), in very tight systems such as clays, shale, as well as in thin films the cross-coupling diffusion coefficient of ions should be derived including the contribution of the diffuse layer. In very tight systems, the pores can function as selective membranes which are partially permeable to some ions.

Although numerical simulation of flow and transport in microscopic charged systems such as microfluidics is feasible, solving the electrokinetics coupled with flow and transport at macroscopic systems such as soils, batteries, and rocks is very challenging, if not infeasible, due to huge discrepancy between the physical scale of diffuse layers versus that of macroscopic porous media, complex nonlinear physical and chemical phenomena, limited computational. Therefore, theoretical upscaling of microscopic phenomena is essential to obtain meaningful macroscopic theories that can be used to model physical problems. However, due to the very complex and highly nonlinear nature of processes in charged porous media, the macroscopic systems at the continuum-scale are only partially understood and our capability to rigorously describe such complex processes is still limited. In Sect. 4, some results derived using hybrid mixture theory and homogenization are discussed. The obtained macroscale equations including generalized forms of Darcy's law and Fick's law are presented, and even though the approaches are quite different, the resulting macroscopic equations are similar.

Upscaling via homogenization is difficult due to the highly varying dependent variables, the nonlinear nature of the microscopic equations, and the uncertainty of capturing all the necessary microscopic physics, but homogenization approaches are becoming more sophisticated and improving. Upscaling via hybrid mixture theory avoids the difficulty of the microscale nonlinear equations, but looses all the microscopic information such as geometry, and it is unclear how macroscopic quantities are related to pore structure.

Overall, the difficulty in modeling such complex media is not only in determining what aspects are crucial, but it is also clear that we do not have a clear understanding of all the physics. Examples provided here include diffusion in bentonite clay and lithium-ion batteries and ionic concentration differences in biological cell membranes.

Acknowledgements We thank the anonymous reviewers and Dr Denis O'Carroll for their fruitful comments and suggestions, in particular comments related to diffusion in charged systems that greatly improved the manuscript. This work was partially supported by the UK Engineering and Physical Sciences Research Council (EPSRC) Grant EP/R021627/1.

Open Access This article is distributed under the terms of the Creative Commons Attribution 4.0 International License (http://creativecommons.org/licenses/by/4.0/), which permits unrestricted use, distribution, and repro- 
duction in any medium, provided you give appropriate credit to the original author(s) and the source, provide a link to the Creative Commons license, and indicate if changes were made.

\section{Appendix: Nomenclature}

Superscripts, Subscripts, and Other Notations

.$\alpha_{j} j$ th component of $\alpha$-phase at macroscale, $\alpha=l, s, b$

. $\alpha \quad \alpha$-Phase on macroscale, $\alpha=l, s, b$

$\sim$ Fluctuation from averaged quantity

$\hat{r}$ Exchange from other interface or phase

${ }^{k, l}$ Difference of the two quantities, i.e., ${ }^{k}-{ }^{l}$

$\left.\cdot\right|_{\alpha_{j}}$ Microscopic property of constituent $j$ in phase [subscript] (non-averaged)

Latin Symbols

$\delta A_{\alpha \beta}$ Portion of $\alpha \beta$-interface in representative elementary volume (REV)

$c^{\alpha_{j}}$ Mass fraction of $j$ th component [mass of $\alpha_{j}$ per mass $\alpha$ ], $c^{\alpha_{j}}=\rho^{\alpha_{j}} / \rho^{\alpha}$ and $\sum_{j=1}^{N} c^{\alpha_{j}}=1$

$c_{b}$ Molar bulk concentration (where the electric potential is zero) at pore scale (mol $\mathrm{m}^{-3}$ )

$c_{i}$ Molar concentration of species $i$ at pore scale $\left(\mathrm{mol} \mathrm{m}^{-3}\right)$

$D_{i}$ Binary diffusion coefficient for ion $i\left(\mathrm{~m}^{2} \mathrm{~s}^{-1}\right)$

$D_{i j}$ Cross-coupled diffusion coefficient for ion $i$ in interaction with ion $j\left(\mathrm{~m}^{2} \mathrm{~s}^{-1}\right)$

$D^{\alpha}$ Electric displacement $\left(\mathrm{C} \mathrm{m}^{-2}\right)$

$e$ Charge of an electron, $4.355 \times 10^{-19}$ coulombs (C)

$\widehat{e}_{\beta}^{\alpha_{j}}$ Rate of mass transfer from phase [subscript] to phase [superscript] per unit mass density $\left(\mathrm{s}^{-1}\right), \widehat{e}_{\beta}^{\alpha}=\sum_{j=1}^{N} C^{\alpha_{j}} \widehat{e}_{\beta}^{\alpha_{j}}$

$\boldsymbol{E}^{\alpha}$ Electric field intensity generated by the charged particles in phase $\alpha\left(\mathrm{V} \mathrm{m}^{-1}\right)$

$\boldsymbol{E}_{\mathrm{T}}$ Total electric field intensity, incorporating an externally applied electric field and that due to charged components $\alpha_{j}\left(\mathrm{~V} \mathrm{~m}^{-1}\right)$

$\boldsymbol{g}$ External supply of momentum (gravity) $\left(\mathrm{m} \mathrm{s}^{-2}\right)$

$\boldsymbol{g}_{I}$ Internal supply of momentum due to fluctuations in $\boldsymbol{\nabla} \boldsymbol{E} \cdot \boldsymbol{E}_{j}$ and $\boldsymbol{\nabla} \boldsymbol{E}_{j} \cdot \boldsymbol{E}\left(\mathrm{m} \mathrm{s}^{-2}\right)$

$G$ Gibbs potential $\left(\mathrm{J} \mathrm{kg}^{-1}\right)$ or $\left(\mathrm{m}^{2} \mathrm{~s}^{-2}\right)$

$\mathbf{j}_{i}$ Microscale molar flux of ions $\left(\mathrm{mol} \mathrm{m}^{-2} \mathrm{~s}^{-1}\right)$

$\boldsymbol{J}^{\alpha_{j}}, \boldsymbol{J}^{\alpha}$ Free current density relative to a fixed (Eulerian) frame of reference $\left(\mathrm{A} \mathrm{m}^{-2}\right)$ $\left(\mathrm{C} \mathrm{m}{ }^{-2} \mathrm{~s}^{-1}\right) \mathcal{J}^{\alpha_{j}}=\boldsymbol{J}^{\alpha_{j}}-q_{e}^{\alpha_{j}} \boldsymbol{v}^{\alpha_{j}}$

$\mathcal{J}^{\alpha_{j}}, \mathcal{J}^{\alpha}$ Free current density of constituent $j$ in phase $\alpha$ and of phase $\alpha$, respectively, measured relative to species $\alpha_{j}, \alpha\left(\mathrm{A} \mathrm{m}^{-2}\right)\left(\mathrm{C} \mathrm{m}^{-2} \mathrm{~s}^{-1}\right)$

$k_{\mathrm{B}}$ Boltzmann constant $=R / N_{a}\left(\mathrm{~J}^{\circ} \mathrm{K}^{-1}\right)$

$n$ Unit normal vector pointing out of the solid phase at the microscale (-)

$\boldsymbol{n}^{\alpha}$ Microscale unit normal vector pointing out of $\alpha$-phase within REV (-)

$N_{a}$ Avogadro's number $\left(\mathrm{mol}^{-1}\right)$

$p$ Pressure $(\mathrm{Pa})$ or $\left(\mathrm{kg} \mathrm{m}^{-1} \mathrm{~s}^{-2}\right)$

$\boldsymbol{P}^{\alpha}$ Polarization density averaged over $\alpha$-phase $\left(\mathrm{C} \mathrm{m}^{-2}\right)$

$P$ Probability of a microscopic state

$q_{e}^{\alpha_{j}}, q_{e}^{\alpha}$ Charge density averaged over $\alpha$-phase $\left(\mathrm{C} \mathrm{m}^{-3}\right)$

$R$ Universal gas constant $\left(\mathrm{J} \mathrm{mol}^{-1}{ }^{\circ} \mathrm{K}^{-1}\right)$

$R_{i}$ Rate of desorption or adsorption of species $i\left(\mathrm{~mol} \mathrm{~m}^{-3} \mathrm{~s}^{-1}\right)$ 
$\widehat{q}^{\alpha_{j}}$ Net rate of charge density gain by species $j$ due to interaction with other species within phase $\alpha$ (does not include that gained to due mass transfer) $\left(\mathrm{C} \mathrm{m}^{-3} \mathrm{~s}^{-1}\right)$

$\widehat{r}^{\alpha_{j}}$ Rate of mass gain due to interaction with other species within the same phase per unit mass density $\left(\mathrm{s}^{-1}\right)$

$t$ Time (s)

$\stackrel{\boldsymbol{t}}{=m}$ Maxwell stress tensor $(\mathrm{Pa})$ or $\left(\mathrm{kg} \mathrm{m}^{-1} \mathrm{~s}^{-2}\right)$

$\underline{\boldsymbol{t}}^{\alpha}$ Total Cauchy stress tensor for the phase $\left(\mathrm{N} \mathrm{m}^{-2}\right)$

${ }^{\bar{T}}$ Temperature $\left({ }^{\circ} \mathrm{K}\right)$

$\widehat{\boldsymbol{T}}_{\beta}^{\alpha}$ Rate of momentum transfer through mechanical interactions from phase [subscript] to phase [superscript] per unit mass density $\left(\mathrm{N} \mathrm{kg}^{-1}\right)$

u Microscale velocity $\left(\mathrm{m} \mathrm{s}^{-1}\right)$

$\boldsymbol{v}^{\alpha_{j}}, \boldsymbol{v}^{\alpha}$ Velocity $\left(\mathrm{m} \mathrm{s}^{-1}\right), \boldsymbol{v}^{\alpha}=\sum_{j=1}^{N} C^{\alpha_{j}} \boldsymbol{v}^{\alpha_{j}}$

$\boldsymbol{v}^{\alpha_{j}, \alpha}$ Diffusion of species $j$ in phase $\alpha=l, s,\left[\mathrm{~m} \mathrm{~s}^{-1}\right], \boldsymbol{v}^{\alpha_{j}, \alpha}=\boldsymbol{v}^{\alpha_{j}}-\boldsymbol{v}^{\alpha}$

$\delta V$ Representative elementary volume (REV)

$\delta V_{\alpha}$ Portion of $\alpha$-phase in REV

$\boldsymbol{w}_{\alpha \beta}^{j}$ Velocity of constituent $j$ at interface between phases $\alpha$ and $\beta\left(\mathrm{m} \mathrm{s}^{-1}\right)$

$W$ Energy of microscopic state $(\mathrm{J})$

$\boldsymbol{x}$ Macroscale spatial variable (m)

$z_{i}$ Valence for species $i$, positive for cations and negative for anions

$z^{\alpha_{j}}$ Charge per unit mass density of constituent $j$ in phase $\alpha\left(\mathrm{C} \mathrm{kg}^{-1}\right), q_{e}^{\alpha_{j}}=\rho^{\alpha_{j}} z^{\alpha_{j}}$

$\widehat{Z}_{\beta}^{\alpha_{j}}, \widehat{Z}_{\beta}^{\alpha}$ Rate of exchange of charge of constituent $j$ from phase $\beta$ to phase $\alpha$ per unit mass $\left(\mathrm{C} \mathrm{kg}^{-1} \mathrm{~s}^{-1}\right)$

\section{Greek Symbols}

$\alpha$ Phase, $\alpha=l$ for liquid, $s$ for solid, and $b$ for bulk (not in porous media)

$\Gamma$ Ionic strength (atoms $\mathrm{m}^{-3}$ )

$\varepsilon^{\alpha}$ Volume fraction of $\alpha$-phase in mesoscale REV (-)

$\epsilon_{0}$ Permittivity in a vacuum. In MKS units, the permittivity has the value of $8.854 \times 10^{-12}$ Farads per meter $\left(\mathrm{F} \mathrm{m}^{-1}\right)$

$\epsilon$ Permittivity $\left(\mathrm{F} \mathrm{m}^{-1}\right)$

$\zeta$ Zeta potential, electric potential measured at the shear plane $(\mathrm{V})$ or $\left(\mathrm{J} \mathrm{C}^{-1}\right)$

$\eta$ Dynamic viscosity (Pa s) or $\left(\mathrm{kg} \mathrm{m}^{-1} \mathrm{~s}^{-1}\right)$

$\lambda_{D}$ Debye length (m)

$\Lambda$ Lagrange multiplier used to enforce charge neutrality (used only if no externally applied electric field) (V)

$\mu^{l_{j}}$ Macroscale chemical potential for species $j$ in the liquid phase $\left(\mathrm{J} \mathrm{kg}^{-1}\right)$ or $\left(\mathrm{m}^{2} \mathrm{~s}^{-2}\right)$

$\mu_{i}$ Microscale chemical potential for species $i$ in the liquid phase $(\mathrm{J})$ or $\left(\mathrm{kg} \mathrm{m}^{2} \mathrm{~s}^{-2}\right)$

$\pi^{l}$ Macroscale swelling potential-change in intensive energy of liquid with respect to porosity while keeping density fixed $(\mathrm{Pa})$ or $\left(\mathrm{kg} \mathrm{m}^{-1} \mathrm{~s}^{-2}\right)$

$\Pi$ Osmotic pressure at microscale $\left(\mathrm{kg} \mathrm{m}^{-1} \mathrm{~s}^{-2}\right)$

$\Pi_{d}$ Differential osmotic pressure at microscale, or difference between osmotic pressure and that at equilibrium $\left(\mathrm{kg} \mathrm{m}^{-1} \mathrm{~s}^{-2}\right)$

$\rho_{e}$ Net charge density $\left(\mathrm{C} \mathrm{m}^{-3}\right)$

$\rho^{j}$ Microscale mass density of $j$ th component [(mass of $\left.j\right) /($ volume of phase)]

$\rho^{\alpha_{j}}$ Partial mass density of $j$ th component of $\alpha$-phase (mass/volume) so that $\varepsilon^{\alpha} \rho^{\alpha_{j}}$ is the total mass of $j$ th constituent in phase $\alpha$ divided by the volume of REV

$\rho^{\alpha}$ Mass density of $\alpha$-phase averaged over $\alpha$-phase [(mass of $\left.\alpha\right) /($ volume of $\alpha)$ ]

$\sigma$ Surface charge density $\left(\mathrm{C} \mathrm{m}^{-2}\right)$ 
$\psi$ Electric potential (V)

$\psi^{\alpha}$ Macroscale Helmholtz potential of phase $\alpha\left(\mathrm{J} \mathrm{kg}^{-1}\right)$ or $\left(\mathrm{m}^{2} \mathrm{~s}^{-2}\right)$

\section{References}

Appelo, C.A.J., Wersin, P.: Multicomponent diffusion modeling in clay systems with application to the diffusion of tritium, iodide, and sodium in opalinus clay. Environ. Sci. Technol. 41(14), 5002-5007 (2007). https:// doi.org/10.1021/es0629256

Appelo, C.A.J., Van Loon, L.R., Wersin, P.: Multicomponent diffusion of a suite of tracers (HTO, CL, BR, I, $\mathrm{Na}, \mathrm{Sr}, \mathrm{Cs}$ ) in a single sample of Opalinus Clay. Geochim. et Cosmochim. Acta 74(4), 1201-1219 (2010)

Arunachalam, H., Onori, S., Battiato, I.: On veracity of macroscopic lithium-ion battery models. J. Electrochem. Soc. 162(10), A1940-A1951 (2015). https://doi.org/10.1149/2.0771509jes

Aziz, R., Joekar-Niasar, V., Martinez-Ferrer, P.: Pore-scale insights into transport and mixing in steady-state two-phase flow in porous media. Int. J. Multiph. Flow 109, 51-62 (2018)

Barclay, L., Thompson, D.: Electron microscopy of sodium montmorillonite. Nature 222, 222-263 (1969)

Bartels, W.B., Mahani, H., Berg, S., Hassanizadeh, S.: Literature review of low salinity waterflooding from a length and time scale perspective. Fuel 236, 338-353 (2019). https://doi.org/10.1016/j.fuel.2018.09. 018. http://www.sciencedirect.com/science/article/pii/S0016236118315527

Basu, S., Sharma, M.M.: Measurement of critical disjoining pressure for dewetting of solid surfaces. J. Colloid Interface Sci. 181(2), 443-455 (1996). https://doi.org/10.1006/jcis.1996.0401

Ben-Yaakov, S.: Diffusion of sea water ions-I. diffusion of sea water into a dilute solution. Geochim. et Cosmochim. Acta 36(12), 1395-1406 (1972)

Bennethum, L.S., Cushman, J.H.: Multicomponent, multiphase thermodynamics of swelling porous media with electroquasistatics: I. Macroscale field equations. Transp. Porous Media 47(3), 309-336 (2002a)

Bennethum, L.S., Cushman, J.H.: Multicomponent, multiphase thermodynamics of swelling porous media with electroquasistatics: II. Constitutive theory. Transp. Porous Media 47(3), 337-362 (2002b)

Bennethum, L.S., Weinstein, T.: Three pressures in porous media. Transp. Porous Media 54(1), 1-34 (2004)

Bergeron, V.: Forces and structure in thin liquid soap films. J. Phys. Condens. Matter 11(19), R215 (1999)

Binks, B.P., Cho, W.G., Fletcher, P.D.I.: Disjoining pressure isotherms for oil-water-oil emulsion films. Langmuir 13(26), 7180-7185 (1997). https://doi.org/10.1021/la970827f

Boudreau, B.P., Meysman, F.J., Middelburg, J.J.: Multicomponent ionic diffusion in porewaters: Coulombic effects revisited. Earth Planet. Sci. Lett. 222(2), 653-666 (2004a)

Boudreau, B.P., Meysman, F.J.R., Middelburg, J.J.: Multicomponent ionic diffusion in porewaters: Coulombic effects revisited. Earth Planet. Sci. Lett. 222(2), 653-666 (2004b)

Bourg, I.C., Bourg, A.C.M., Sposito, G.: Modeling diffusion and adsorption in compacted bentonite: a critical review. J. Contam. Hydrol. 61(1-4), 293-302 (2003)

Bradbury, M.H., Baeyens, B.: Porewater chemistry in compacted re-saturated MX-80 bentonite. J. Contam. Hydrol. 61(1-4), 329-338 (2003)

Butt, H.-J., Butt, H.J.B., Graf, K., Kappl, M.: Physics and Chemistry of Interfaces, 3rd edn. Wiley, New York (2013)

Choi, W., Sharma, A., Qian, S., Lim, G., Joo, S.W.: Is free surface free in micro-scale electrokinetic flows? J. Colloid Interface Sci. 347(1), 153-155 (2010). https://doi.org/10.1016/j.jcis.2010.03.049

Churaev, N., Sobolev, V.: Prediction of contact angles on the basis of the Frumkin-Derjaguin approach. Adv. Colloid Interface Sci. 61, 1-16 (1995). https://doi.org/10.1016/0001-8686(95)00257-q

Ciucci, F., Lai, W.: Derivation of micro/macro lithium battery models from homogenization. Transp. Porous Media 88(2), 249-270 (2011). https://doi.org/10.1007/s11242-011-9738-5

Coleman, B.D., Noll, W.: The thermodynamics of elastic materials with heat conduction and viscosity. Arch. Rational Mech. Anal. 13, 167-178 (1963)

Cushman, J.H., Bennethum, L.S., Hu, B.X.: Primer on upscaling tools for porous media. Adv. Water Resour. 25(8-12), 1043-1067 (2002)

Davis, J.A., James, R.O., Leckie, J.O.: Surface ionization and complexation at the oxide/water interface: I. Computation of electrical double layer properties in simple electrolytes. J. Colloid Interface Sci. 63(3), 480-499 (1978)

Dimitrova, T.D., Leal-Calderon, F., Gurkov, T.D., Campbell, B.: Disjoining pressure vs thickness isotherms of thin emulsion films stabilized by proteins. Langmuir 17(26), 8069-8077 (2001). https://doi.org/10. 1021/la0111147

Eringen, A.C., Maugin, G.A.: Electrodynamics of Continua I. Springer, New York (1990) 
Farajzadeh, R., Andrianov, A., Krastev, R., Hirasaki, G., Rossen, W.: Foam-oil interaction in porous media: implications for foam assisted enhanced oil recovery. Adv. Colloid Interface Sci. 183-184, 1-13 (2012). https://doi.org/10.1016/j.cis.2012.07.002

Feng, L., Jia, Y., Chen, X., Li, X., An, L.: A multiphasic model for the volume change of polyelectrolyte hydrogels. J Chem Phys 133(11), 114,904 (2010). https://doi.org/10.1063/1.3484236

García-Gutiérrez, M., Cormenzana, J.L., Missana, T., Mingarro, M.: Diffusion coefficients and accessible porosity for HTO and 36Cl in compacted FEBEX bentonite. Appl. Clay Sci. 26(1-4), 65-73 (2004)

Gilson, M.K., Davis, M.E., Luty, B.A., McCammon, J.A.: Computation of electrostatic forces on solvated molecules using the Poisson-Boltzmann equation. J. Phys. Chem. 97(14), 3591-3600 (1993)

Glaus, M.A., Baeyens, B., Bradbury, M.H., Jakob, A., Van Loon, L.R., Yaroshchuk, A.: Diffusion of ${ }^{22}$ Na and ${ }^{85} \mathrm{Sr}$ in montmorillonite: evidence of interlayer diffusion being the dominant pathway at high compaction. Environ. Sci. Technol. 41(2), 478-485 (2007)

Goldberg, S.: Use of Surface Complexation Models in Soil Chemical Systems, vol. 47, pp. 233-329. Academic Press, London (1992)

Goldberg, S.: Application of surface complexation models to anion adsorption by natural materials. Environ. Toxicol. Chem. 33(10), 2172-2180 (2014)

Grasso, D., Subramaniam, K., Butkus, M., Strevett, K., Bergendahl, J.: A review of non-DLVO interactions in environmental colloidal systems. Rev. Environ. Sci. Biotechnol. 1(1), 17-38 (2002)

Guijt, R.M., Baltussen, E., van der Steen, G., Schasfoort, R.B.M., Schlautmann, S., Billiet, H.A.H., Frank, J., van Dedem, G.W.K., van den Berg, A.: New approaches for fabrication of microfluidic capillary electrophoresis devices with on-chip conductivity detection. Electrophoresis 22(2), 235-241 (2001)

Gupta, A., Stone, H.A.: Electrical double layers: effects of asymmetry in electrolyte valence on steric effects, dielectric decrement, and ion-ion correlations. Langmuir 34(40), 11971-11985 (2018)

Guyton, A., Hall, J.: Textbook of Medical Physiology. W.B. Saunders Company, New York (1996)

Hassanizadeh, S.M., Gray, W.G.: Mechanics and thermodynamics of multiphase flow in porous media including interphase boundaries. Adv. Water Resour. 13, 169-186 (1990)

Hiemstra, T., Riemsdijk, W.V.: A surface structural approach to ion adsorption: the charge distribution (CD) model. J. Colloid Interface Sci. 179(2), 488-508 (1996)

Hill, T.L.: An Introduction to Statistical Thermodynamics. Dove Publications, Ltd, Auckland (1960)

Hueckel, T.A.: Water-mineral interaction in hygromechanics of clays exposed to environmental loads: a mixture-theory approach. Can. Geotech. J. 29(6), 1071-1086 (1992)

Hunter, R.: Zeta Potential in Colloid Science: Principles and Applications. Colloid Science: A Series of Monographs. Academic Press, London (1988)

Hunter, R.: Foundations of Colloid Science, vol. 2. Clarendon Press, Oxford (1989)

Huyghe, J.M., Janssen, J.D.: Quadriphasic mechanics of swelling incompressible porous media. Int. J. Eng. Sci. 35, 793-802 (1997)

Huyghe, J.M., Wilson, W., Malakpoor, K.: On the thermodynamical admissibility of the triphasic theory of charged hydrated tissues. ASME J. Biomech. Eng. 131, 044,504 (2009)

Ignarro, L.: Nitric Oxide: Biology and Pathobiology. Academic Press, San Diego (2000)

Israelachvili, J., Pashley, R.: The hydrophobic interaction is long range, decaying exponentially with distance. Nature 300(5890), 341-342 (1982)

Joekar-Niasar, V., Mahani, H.: Nonmonotonic pressure field induced by ionic diffusion in charged thin films. Ind. Eng. Chem. Res. 55(21), 6227-6235 (2016). https://doi.org/10.1021/acs.iecr.6b00842

Karraker, K., Radke, C.: Disjoining pressures, zeta potentials and surface tensions of aqueous non-ionic surfactant/electrolyte solutions: theory and comparison to experiment. Adv. Colloid Interface Sci. 96(1), 231-264 (2002). A Collection of Papers in Honour of Nikolay Churaev on the Occasion of his 80th Birthday

Kilic, M.S., Bazant, M.Z., Ajdari, A.: Steric effects in the dynamics of electrolytes at large applied voltages: I. Double-layer charging. Phys. Rev. E 75, 021502 (2006)

Kilic, M.S., Bazant, M.Z., Ajdari, A.: Steric effects in the dynamics of electrolytes at large applied voltages. II. Modified Poisson-Nernst-Planck equations. Phys. Rev. E Stat. Nonlinear Soft Matter Phys. 75(2.1), 021,503 (2007)

Kirby, B.J.: Micro-and Nanoscale Fluid Mechanics: Transport in Microfluidic Devices. Cambridge University Press, Cambridge (2010)

Kirby, B.J., Hasselbrink, E.F.: Zeta potential of microfluidic substrates: 1. Theory, experimental techniques, and effects on separations. Electrophoresis 25(2), 1522-2683 (2004)

Kornyshev, A.A.: Double-layer in ionic liquids: paradigm change? J. Phys. Chem. B 111(20), 5545-5557 (2007)

Kozaki, T., Fujishima, A., Saito, N., Sato, S., Ohashi, H.: Effects of dry density and exchangeable cations on the diffusion process of sodium ions in compacted montmorillonite. Eng. Geol. 81(3), 246-254 (2005) 
Lager, A., Webb, K., Black, C., Singleton, M., Sorbie, K.: Low salinity oil recovery-an experimental investigation. In: International Symposium of the Society of Core Analysts, Trondheim, Norway, pp. SCA2006-36 (2006)

Lasaga, A.C.: The treatment of multi-component diffusion and ion pairs in diagenetic fluxes. Am. J. Sci. 279(10), 324-346 (1079)

Lehikoinen, J., Carlsson, T., Muurinen, A., Olin, M., Salonen, P.: Evaluation of factors affecting diffusion in compacted bentonite. In: MRS Online Proceedings Library Archive, vol. 412 (1995)

Le, T., Moyne, C., Murad, M.: A three-scale model for ionic solute transport in swelling clays incorporating ion-ion correlation effects. Adv. Water Resour. 75, 31-52 (2015)

Leroy, P., Revil, A.: A triple-layer model of the surface electrochemical properties of clay minerals. J. Colloid Interface Sci. 270(2), 371-380 (2004)

Leroy, P., Revil, A., Coelho, D.: Diffusion of ionic species in bentonite. J. Colloid Interface Sci. 296(1), 248-55 (2006). https://doi.org/10.1016/j.jcis.2005.08.034

Ligthelm, D., Gronsveld, J., Hofman, J., Brussee, N., Marcelis, F., van der Linde, H.A.: Novel waterflooding strategy by manipulation of injection brine composition. In: SPE EUROPEC/EAGE Annual, p. SPE 119835 (2009)

Lyklema, J.: Fundamentals of Interface and Colloid Science, vol. 2. Elsevier, Amsterdam (1995)

Mahani, H., Berg, S., Ilic, D., Bartels, W.B., Joekar-Niasar, V.: Kinetics of low-salinity-flooding effect. SPE J. 20, 8-20 (2015)

Malaterre, V., Ogorka, J., Loggia, N., Gurny, R.: Oral osmotically driven systems: 30 years of development and clinical use. Eur. J. Pharm. Biopharm. 73(3), 311-323 (2009). https://doi.org/10.1016/j.ejpb.2009. 07.002

Manica, R., Connor, J.N., Carnie, S.L., Horn, R.G., Chan, D.Y.C.: Dynamics of interactions involving deformable drops: hydrodynamic dimpling under attractive and repulsive electrical double layer interactions. Langmuir 23(2), 626-637 (2007). https://doi.org/10.1021/la0620760. PMID: 17209614

McGuire, P.L., Chatham, J.R., Paskvan, F.K., Sommer, D.M., Carini, F.H.: Low salinity oil recovery: an exciting new EOR opportunity for Alaska's North Slope. In: SPE Western Regional Meeting, 30 March-1 April, Irvine, California (SPE93903) (2005)

Mikelonis, A., Youn, S., Lawler, D.: DLVO approximation methods for predicting the attachment of silver nanoparticles to ceramic membranes. Langmuir 32, 1723-1731 (2016)

Moyne, C., Murad, M.A.: A two-scale model for coupled electro-chemo-mechanical phenomena and Onsager's reciprocity relations in expansive clays: I Homogenization analysis. Transp. Porous Media 62(3), 333380 (2006)

Muurinen, A., Karnland, O., Lehikoinen, J.: Effect of homogenization on the microstructure and exclusion of chloride in compacted bentonite. Phys. Chem. Earth Parts A/B/C 32(1-7), 485-490 (2007)

Naidu, R., Bolan, N., Kookana, R.S., Tiller, K.: Ionic-strength and pH effects on the sorption of cadmium and the surface charge of soils. Eur. J. Soil Sci. 45(4), 419-429 (1994)

Newman, J., Thomas-Alyea, K.: Electrochem. Syst. Wiley, Hoboken (2004)

Newman, J., Tiedemann, W.: Porous-electrode theory with battery applications. AIChE J. 21(1), 25-41 (1975)

Nicolini, J.V., Ferraz, H.C., Borges, C.P.: Effect of seawater ionic composition modified by nanofiltration on enhanced oil recovery in Berea sandstone. Fuel 203, 222-232 (2017)

Norde, W., Lyklema, J.: The adsorption of human plasma albumin and bovine pancreas ribonuclease at negatively charged polystyrene surfaces: I. Adsorption isotherms. Effects of charge, ionic strength, and temperature. J. Colloid Interface Sci. 66(2), 257-265 (1978)

Ohshima, H.: The Derjaguin-Landau-Verwey-Overbeek (DLVO) Theory of Colloid Stability. Wiley, New York (2012). https://doi.org/10.1002/9781118135440.ch3

Onsager, L.: Reciprocal relations in irreversible processes. I. Phys. Rev. 37, 405-426 (1931a)

Onsager, L.: Reciprocal relations in irreversible processes. II. Phys. Rev. 38, 2265-2279 (1931b)

Onsager, L.: The effects of shape on the interaction of colloidal particles. Ann. N. Y. Acad. Sci. 51(1), 627-659 (1949)

Pusch, R.: Waste disposal in rock. Dev. Geotech. Eng. 76, 354 (1994)

Pusch, R., Yong, R.N., Nakano, M.: Containment of High-Level Radioactive and Hazardous Solid Wastes with Clay Barriers. CRC Press, Boca Raton (2007)

Revil, A., Linde, N.: Chemico-electromechanical coupling in microporous media. J. Colloid Interface Sci. 302(2), 682-694 (2006)

Richardson, G., Denuault, G., Please, C.P.: Multiscale modelling and analysis of lithium-ion battery charge and discharge. J. Eng. Math. 72(1), 41-72 (2012). https://doi.org/10.1007/s10665-011-9461-9

Rio, J.A., Whitaker, S.: Maxwell's equations in two-phase systems I: local electrodynamic equilibrium. Transp. Porous Media 39, 159-186 (2000) 
Rivet, S., Lake, L.W., Pope, G.A.: A coreflood investigation of low-salinity enhanced oil recovery. In: SPE Annual Technical Conference and Exhibition (2010). https://doi.org/10.2118/134297-ms

Rubi, J., Kjelstrup, S.: Mesoscopic nonequilibrium thermodynamics gives the same thermodynamic basis to Butler-Volmer and Nernst equations. J. Phys. Chem. B 107(48), 13471-13477 (2003)

Schmuck, M., Bazant, M.: Homogenization of the Poisson-Nernst-Planck equations for ion transport in charged porous media. SIAM J. Appl. Math. 75(3), 1369-1401 (2015)

Sedighi, M., Thomas, H.R.: Micro porosity evolution in compacted swelling clays-a chemical approach. Appl. ied Clay Sci. 101, 608-618 (2014)

Sedighi, M., Thomas, H.R., Al Masum, S., Vardon, P.J., Nicholson, D., Chen, Q.: Geochemical modelling of hydrogen gas migration in an unsaturated bentonite buffer. Geol. Soc. Lond. Spec. Publ. 415(1), 189-201 (2015)

Sedighi, M., Thomas, H., Vardon, P.: Reactive transport of chemicals in compacted bentonite under nonisothermal water infiltration. ASCE J. Geotech. Geoenviron. Eng. 144(10), 04018 (2018)

Siepmann, J., Siepmann, F.: Mathematical modeling of drug delivery. Int. J. Pharm. 364, 328-343 (2008)

Sohrabi, M., Mahzari, P., Farzaneh, S.A., Mills, J.R., Tsolis, P., Ireland, S.: Novel insights into mechanisms of oil recovery by use of low-salinity-water injection. SPE J. 22(02), 407-416 (2017)

Trefalt, G., Behrens, S.H., Borkovec, M.: Charge regulation in the electrical double layer: ion adsorption and surface interactions. Langmuir 32, 380-400 (2016)

Van Loon, L.R., Glaus, M.A., Müller, W.: Anion exclusion effects in compacted bentonites: towards a better understanding of anion diffusion. Appl. Geochem. 22(11), 2536-2552 (2007)

van Oss, C.J., Giese, R.F., Costanzo, M.: DLVO and non-DLVO interactions in hectorite. Clays Clay Miner. 38(2), 151-159 (1990)

Weinstein, T., Bennethum, L.S., Cushman, J.H.: Two-scale, three-phase theory for swelling drug delivery systems. Part I: constitutive theory. J. Pharm. Sci. 97, 1878-1903 (2008a)

Weinstein, T., Bennethum, L.S., Cushman, J.H.: Two-scale, three-phase theory for swelling drug delivery systems. Part II: flow and transport models. J. Pharm. Sci. 97, 1904-1915 (2008b)

Wersin, P., Curti, E., Appelo, C.A.J.: Modelling bentonite-water interactions at high solid/liquid ratios: swelling and diffuse double layer effects. Appl. Clay Sci. 26(1-4), 249-257 (2004)

Yi, Y., Sun, J., Lu, Y., Liao, Y.: Programmable and on-demand drug release using electrical stimulation. Biomicrofluidics 9, 022401 (2015)

Yong, R., Nakano, M., Pusch, R.: Environmental Soil Properties and Behaviour. CRC Press, Boston (2012)

Yong, R., Pusch, R., Nakano, M.: Containment of High-Level Radioactive and Hazardous Solid Wastes with Clay Barriers. CRC Press, Boston (2010)

Publisher's Note Springer Nature remains neutral with regard to jurisdictional claims in published maps and institutional affiliations. 